\title{
Isocyanic acid (HNCO) in the hot molecular core G331.512-0.103: observations and chemical modelling
}

\author{
Carla M. Canelo ${ }^{\circledR},{ }^{1 \star}$ Leonardo Bronfman, ${ }^{2 \star}$ Edgar Mendoza, ${ }^{1,3 \star}$ Nicolas Duronea, ${ }^{4}$ Manuel Merello ${ }^{\circledR}, 2$ \\ Miguel Carvajal ${ }^{\oplus}, 5,6$ Amâncio C. S. Friaça ${ }^{1}$ and Jacques Lepine ${ }^{1}$ \\ ${ }^{1}$ Departamento de Astronomia, Instituto de Astronomia, Geofísica e Ciências Atmosféricas, Universidade de São Paulo, São Paulo, 05508-090, Brazil \\ ${ }^{2}$ Departamento de Astronomía, Universidad de Chile, Casilla 36-D, Santiago de Chile, Chile \\ ${ }^{3}$ Observatório do Valongo, Universidade Federal do Rio de Janeiro, Ladeira Pedro Antônio, 43, Rio de Janeiro, RJ 20.080-090, Brazil \\ ${ }^{4}$ Instituto de Astrofísica de La Plata (UNLP - CONICET), La Plata, Argentina \\ ${ }^{5}$ Dept. Ciencias Integradas, Facultad de Ciencias Experimentales, Centro de Estudios Avanzados en Física, Matemática y Computación, Unidad Asociada \\ GIFMAN, CSIC-UHU, Universidad de Huelva, Spain \\ ${ }^{6}$ Instituto Universitario Carlos I de Física Teórica y Computacional, Universidad de Granada, Spain
}

Accepted 2021 April 20. Received 2021 April 20; in original form 2021 January 27

\begin{abstract}
Isocyanic acid (HNCO) is a simple molecule with a potential to form prebiotic and complex organic species. Using a spectral survey collected with the Atacama Pathfinder EXperiment, in this work we report the detection of 42 transitions of HNCO in the hot molecular core/outflow G331.512-0.103 (hereafter G331). The spectral lines were observed in the frequency interval $\sim 160-355 \mathrm{GHz}$. By means of Local Thermodynamic Equilibrium analyses, applying the rotational diagram method, we studied the excitation conditions of HNCO. The excitation temperature and column density are estimated to be $T_{e x}=58.8 \pm 2.7 \mathrm{~K}$ and $N=(3.7 \pm 0.5) \times 10^{15} \mathrm{~cm}^{-2}$, considering beam dilution effects. The derived relative abundance is between $(3.8 \pm 0.5) \times 10^{-9}$ and $(1.4 \pm 0.2) \times 10^{-8}$. In comparison with other hot molecular cores, our column densities and abundances are in agreement. An update of the internal partition functions of the four CHNO isomers: HNCO; cyanic acid, HOCN; fulminic acid, HCNO; and isofulminic acid, HONC is provided. We also used the astrochemical code NAUTILUS to model and discuss HNCO abundances. The simulations could reproduce the abundances with a simple zero-dimensional model at a temperature of $60 \mathrm{~K}$ and for a chemical age of $\sim 10^{5} \mathrm{yr}$, which is larger than the estimated dynamical age for G331. This result could suggest the need for a more robust model and even the revision of chemical reactions associated with HNCO.
\end{abstract}

Key words: astrochemistry-molecular data-molecular processes - methods: observational - ISM: molecules.

\section{INTRODUCTION}

The isocyanic acid (HNCO) is the simplest molecule containing four of the six biogenic elements: carbon, hydrogen, oxygen, nitrogen, phosphorus, and sulphur (abbreviated as CHONPS) that are present in all living beings. Although it has only four atoms, HNCO could be precursor of other prebiotic and complex organic molecules $\left(\mathrm{COMs}^{1}\right)$, which are of great astrochemical and astrobiological interest due to their potential to form molecules such as amino acids, sugars, and nucleobases (e.g. Gorai et al. 2020, and references therein). HNCO molecules are included as a peptide bond $[-(\mathrm{H}) \mathrm{N}$ $\mathrm{C}(\mathrm{O})$-] between any two single amino acid, such as glycine (Fedoseev et al. 2015). Moreover, recent experiments, simulating interstellar ice chemical processes, have shown that $\mathrm{HNCO}$ and $\mathrm{CH}_{4}$ can form molecules with peptide bonds in solid state under far-ultraviolet

\footnotetext{
^E-mail: camcanelo@gmail.com (CMC); leo@das.uchile.cl (LB); emendoza@astro.ufrj.br (EM)

${ }^{1}$ As discussed in Herbst \& van Dishoeck (2009), the term complex is labelled by astronomers if not by chemist, and makes a reference to interstellar molecules with six or more atoms including the element carbon.
}

(UV) irradiation (Ligterink et al. 2018). The observation of COMs in astrophysical environments, along with the study of their formation and destruction pathways, are important for a better comprehension of the origins of life on Earth and elsewhere.

The potential interest of HNCO in radio astronomy has generated and stimulated debates, specially about its formation and detection. Among the first experimental works, Jones et al. (1950) and Jones $\&$ Badger (1950) made experimental investigations of the infrared and microwave spectra of HNCO and HNCS. Hocking, Gerry \& Winnewisser $(1972,1975)$ carried out laboratory measurements to study the HNCO microwave and millimetre spectrum. Concurrently, Snyder \& Buhl (1972) reported the results of a survey for HNCO in Galactic molecular sources, concluding about its detection in Sgr B2. HNCO is a near prolate asymmetric top molecule with a linearity barrier at $1899 \mathrm{~cm}^{-1}$ (Niedenhoff et al. 1995); its rotational levels undergo a hyperfine splitting caused by the nuclear spin of nitrogen (Lapinov et al. 2007). The rotational levels of HNCO are assigned by the spectroscopic notation for the asymmetric top, such as $J_{K_{a}, K_{c}}$, where $J$ is the rotational angular momentum, and $K_{a}$ and $K_{c}$ are the projections of $J$ to the molecule fixed principal $a$ - and $c$-axes, respectively (Hocking, Gerry \& Winnewisser 1974; Niedenhoff et al. 1995; Zinchenko, Henkel \& Mao 2000). 
Churchwell et al. (1986) found that the most likely excitation mechanism of $\mathrm{HNCO}$ in Sgr B2 might be radiative rather than collisional, so the molecule can be an optimal tracer of the farinfrared field (at $\lambda \approx 110$ and $330 \mu \mathrm{m}$ ). As a consequence of the energy structure and short lifetimes of the excited $K_{a}$ ladders of HNCO, its populational diagram can be substantially induced by far-infrared radiation. In a study based on observations of HNCO $\left(5_{0,5}-4_{0,4}\right)$ in massive star-forming regions, Li et al. (2013) found that the HNCO emission show signs of embedded mid-infrared or far-infrared sources, and also a correlation with emission of $\mathrm{HC}_{3} \mathrm{~N}$.

As we mentioned previously, the first detection of $\mathrm{HNCO}$ in the interstellar medium (ISM) was towards the molecular cloud complex Sgr B2 (Snyder \& Buhl 1972). Since then, HNCO has been periodically reported in several astronomical objects, from comets up to external galaxies. Based on sub-mm observations of comets, Biver et al. (2006) carried out a comparative study of molecules such as $\mathrm{CH}_{3} \mathrm{OH}, \mathrm{H}_{2} \mathrm{CO}, \mathrm{HNCO}$, among others, towards the comets C/1999 T1 (McNaught-Hartley), C/2001 A2 (LINEAR), C/2000 WM1 (LINEAR), and 153P/Ikeya-Zhang. Lis et al. (1997) performed observations of Comet C/1996 B2 (Hyakutake) and reported for the first time the $\mathrm{HNCO} 16_{0,16}-15_{0,15}$ transition, at $v \approx 351633.257 \mathrm{MHz}$, in a comet. Crovisier (1998) discussed the presence of HNCO in comets Hyakutake and Hale-Bopp; its synthesis could be associated with the $\mathrm{OCN}^{-}$anion. In the context of planetary atmospheres, based on Herschel/HIFI-PACS and Cassini/CIRS studies of Titan's atmosphere, Dobrijevic et al. (2014) carried out photochemical models to predict the presence of compounds such as $\mathrm{NO}, \mathrm{HNCO}$, and $\mathrm{N}_{2} \mathrm{O}$. In low-mass protostellar objects, $\mathrm{HNCO}$ was studied along with $\mathrm{NH}_{2} \mathrm{CHO}$ in solar-type protostars and protostellar shocks (Mendoza et al. 2014; LópezSepulcre et al. 2015, 2019).

More recently, Hernández-Gómez et al. (2019) observed HNCO towards IRAS 16293-2422 and computed models using the astrochemical code NAUTILUS. Their study included observations from several telescopes including the Atacama Pathfinder Experiment $(A P E X)$. Considering evolved objects, HNCO was observed in the oxygen-rich circumstellar envelope around an intermediate-mass evolved star, $\mathrm{OH} 231.8+4.2$, which harbours a bipolar molecular outflow (Velilla Prieto et al. 2015). In the context of high-mass star-forming regions, Zinchenko et al. (2000) surveyed 81 dense high-mass star-forming regions and emission lines of HNCO were detected towards 57 sources, with a tentative detection of $\mathrm{HN}^{13} \mathrm{CO}$ $\left(10_{0,10}-9_{0,9}\right)$ at $\sim 220 \mathrm{GHz}$ in G310.12-0.20. Gorai et al. (2020) carried out a study of $\mathrm{HNCO}, \mathrm{NH}_{2} \mathrm{CHO}$ and $\mathrm{CH}_{3} \mathrm{NCO}$ in the hot molecular core $\mathrm{G} 10.47+0.03$, finding that they are chemically linked with each other according to their data analysis results and chemical models. In the same source, Wyrowski, Schilke \& Walmsley (1999) had previously reported the first detection of vibrationally excited $\mathrm{HNCO}$, as part of a study that was mainly focused on $\mathrm{HC}_{3} \mathrm{~N}$. In Sgr B2 and Orion KL, Turner (1991) analysed several transitions of HNCO in the frequency interval $\sim 87-220 \mathrm{GHz}$. In extragalactic sources, Nguyen-Q-Rieu et al. (1991) reported the first detection of HNCO in external galaxies. They observed the $4_{0,4}-3_{0,3}$, and $6_{0,6^{-}}$ $5_{0,5}$ transitions towards NGC 253 using the IRAM-30 m observatory. Meier \& Turner (2005) observed emission of HNCO towards the nearby spiral galaxy IC 342, finding a correlation with another tracer as $\mathrm{CH}_{3} \mathrm{OH}$. Martín et al. (2006) analysed the physical conditions of HNCO, among other chemical species, using a $2 \mathrm{~mm}$ spectral survey towards NGC 253. Martín, Martín-Pintado \& Mauersberger (2009) determined HNCO abundances in galaxies, concluding that $\mathrm{HNCO}$ is a good tracer to diagnose the evolutionary state of nuclear starburst.
G331 has proven to be very rich source in molecular emission (e.g. Bronfman et al. 2008; Merello et al. 2013b; Mendoza et al. 2018; Duronea et al. 2019; Hervías-Caimapo et al. 2019) and the analysis of HNCO could be an important tool not only to understand the physical properties of the source but also to complement the inventory of Galactic sources where this molecule has been detected. Moreover, the present study could increase the chemical knowledge of the molecular species and their reaction networks present in G331.

In this work, we focus on the identification of HNCO in the hot molecular core G331, which is one of the most luminous and energetic outflows known in the Galaxy. It is located towards the brightest and most massive dust condensation of the star-forming region of G331.5-0.1, placed in the Norma spiral arm at a heliocentric distance of approximately $7.5 \mathrm{kpc}$ (Merello et al. 2013a). The mass of the core is about $40 \mathrm{M}_{\odot}$ while the mass of each outflow lobe is about $25 \mathrm{M}_{\odot}$ (Hervías-Caimapo et al. 2019). G331 also presents a momentum of $\sim 2.4 \times 10^{3} \mathrm{M}_{\odot} \mathrm{km} \mathrm{s}^{-1}$ and a kinetic energy of $\sim 1.4 \times 10^{48} \mathrm{erg}$ (Bronfman et al. 2008). Such values are expected in flows driven by young massive stellar objects with $\mathrm{L}_{b o l} \sim 1 \times 10^{5} L_{\odot}$ (e.g. Bally 2016). Observations in the ALMA band 7 receiver revealed a main hydrogen density of the core of $\sim 5 \times 10^{6} \mathrm{~cm}^{-3}$ and a temperature around $70 \mathrm{~K}$ (Hervías-Caimapo et al. 2019). Moreover, the observed $\mathrm{SiO}$ and $\mathrm{SO}_{2}$ molecular lines also traced a denser region with temperatures up to $200 \mathrm{~K}$, which suggests an expanding shelllike structure.

Another approach to understand the chemical network of HNCO in G331 is to simulate its abundance with an astrochemical model. The evidence of ammonium salts in comet 67P/Churyumov-Gerasimenko can suggest that species like halides and HNCO are in the form of salt, presenting high sublimation temperatures which would not allow their detection in the gas phase and could explain the relatively low interstellar abundances of HNCO (Altwegg et al. 2020). In this sense, the NAUTILUs code (Ruaud, Wakelam \& Hersant 2016) considers both gas and grain reactions to perform time-dependent simulations of molecular abundances in hot and cold molecular cores, which can allow an overview of the HNCO chemistry in G331.

The article is divided as following: Section 2 presents the methodology of the observations and data analysis; Section 3 exhibits the detected lines and their physical analysis; these results are also discussed in Section 4 together with the NAUTILUS simulations; and finally, the summary and conclusions of this work are presented in Section 5 .

\section{METHODOLOGY}

The observations have been obtained with the APEX telescope ${ }^{2}$ (Güsten et al. 2006) using the single point mode towards the coordinates RA:DEC $=16^{\mathrm{h}} 12^{\mathrm{m}} 10.1^{\mathrm{s}},-51^{\circ} 28^{\prime} 38.1^{\prime \prime}$. The spectral line setups were collected over various semesters between 2014 and 2019. We have used APEX-1 and APEX-2 receivers of the Swedish Heterodyne Facility Instrument (SHeFI; Vassilev et al. 2008) to observe bands within the intervals $213-275 \mathrm{GHz}$ and 267-378 GHz, respectively. As backend, the eXtended bandwidth Fast Fourier Transform Spectrometer2 has been used. The spectral

\footnotetext{
${ }^{2}$ This publication is based on data acquired with the APEX, over various semesters between 2014 and 2019, under projects IDs C-094.F-9709B-2014, C-097.F-9710A-2016, C-099.F-9702A-2017, C-0102.F-9702B-2018, and C0104.F-9703B-2019. APEX is a collaboration between the Max-PlanckInstitut fur Radioastronomie, the European Southern Observatory, and the Onsala Space Observatory.
} 
resolution, corresponding to a velocity resolution, has been obtained between $\sim 0.15$ and $0.25 \mathrm{~km} \mathrm{~s}^{-1}$ for a noise level of $\sim 30 \mathrm{mK}$. This paper includes results from the last observations conducted with the SEPIA B5 receiver (Belitsky et al. 2018), since various lines of $\mathrm{HNCO}$ were discovered within the frequency interval covered by that receiver (159-211 GHz). For further clarity, the resolution of the spectra exhibited in the present work has been degraded to a common value of $1 \mathrm{~km} \mathrm{~s}^{-1}$. The original intensity, obtained in scale of antenna temperature $\left(T_{\mathrm{a}}\right)$, has been converted to the main-beam temperature ( $T_{\mathrm{mb}}$ ) scale using main-beam efficiency values of $\eta_{\mathrm{mb}} \approx 0.80,0.75$, and 0.73 at the frequencies 208, 230, and $352 \mathrm{GHz},{ }^{3}$ respectively, whose Half Power Beam Width values are within $\sim 17-30$ arcsec.

The data reduction and line identification have been carried out using the CLASS/GILDAS ${ }^{4}$ and CASSIS $^{5}$ softwares. Lines have been analysed using Spectroscopic databases such as the NIST ${ }^{6}$ Recommended Rest Frequencies for Observed Interstellar Molecular Microwave Transitions (Lovas 2004), $\mathrm{CDMS}^{7}$ (Endres et al. 2016), JPL $^{8}$ (Pickett et al. 1998) and Splatalogue. ${ }^{9}$ The radiative analyses have been carried out assuming Local Thermodynamic Equilibrium (LTE), excitation temperatures, and column densities were estimated from rotational diagrams (Goldsmith \& Langer 1999). The rotational diagrams were constructed using CASSIS, which requires a given calibration uncertainty to compute rotational temperatures and column densities with their respective errors. A calibration uncertainty of 30 per cent (Dumke \& Mac-Auliffe 2010) was considered in this work. Descriptions on the methodology and data analysis have been presented in previous works (e.g. Mendoza et al. 2018; Duronea et al. 2019).

\section{RESULTS}

In general, the spectral lines of HNCO have been identified over the $3 \sigma$ level. Lines with ladders $K_{a}=0,1$, and 2 have been detected and independently analysed in Section 3.1. Across the different $K_{a}$-ladder lines of $\mathrm{HNCO}$, it is noted that some of them are more sensitive to the presence of the outflow. In order to illustrate that, and considering the case of HNCO lines affected by an outflow in the circumstellar envelope of the late star OH 231.8+4.2 (Velilla Prieto et al. 2015), we examine in detail two HNCO lines in G331.

We obtained Gaussian fit parameters for the identified HNCO lines. The results are presented in Table 1, where we list both the spectroscopic and fit parameters with their respective uncertainties. Concerning the HNCO line intensities and integrated areas, their values decrease inversely with the $K_{a}$ ladder number, as it was also discussed in previous observational studies (Churchwell et al. 1986; Zinchenko et al. 2000; Velilla Prieto et al. 2015). While the HNCO $K_{a}=0$ lines are strong enough to be detected, those with $K_{a}=2$ require a more carefully analysis due to the weakness of the emission. We have performed the line identification adopting three standard criteria (e.g. Snyder et al. 2005), namely: (a) the agreement between the rest frequency of an assigned transition with the frequency at the local source rest velocity of the source, which for G331 is $V_{l s r} \simeq-90 \mathrm{~km} \mathrm{~s}^{-1}$; (b) lines with signal-to-noise ratio over

\footnotetext{
${ }^{3}$ http://www.apex-telescope.org/telescope/efficiency/

${ }^{4} \mathrm{https} / / /$ www.iram.fr/IRAMFR/GILDAS/

${ }^{5}$ http://cassis.irap.omp.eu/

${ }^{6}$ http://physics.nist.gov/cgi-bin/micro/table5/start.pl

${ }^{7}$ https://www.astro.uni-koeln.de/cdms/catalog

${ }^{8}$ https://spec.jpl.nasa.gov/

${ }^{9}$ https://www.cv.nrao.edu/php/splat/
}

the limit of detection $(>3 \sigma)$; (c) considering the relative intensities of predicted lines, e.g. under LTE conditions, and comparing with previous observational studies.

\subsection{Qualitative analysis}

\subsubsection{HNCO $\mathrm{K}_{\mathrm{a}}=0$}

Nine lines of HNCO $K_{a}=0$ have been detected and are exhibited in Fig. 1. They have been identified by selecting and superposing only HNCO $K_{a}=0$ transitions over the spectral survey. The line intensities of those transitions are the highest in comparison to the $K_{a}=1,2$, and even 3 ladders. Examining the spectra, an antenna temperature value around $0.6 \mathrm{~K}$ could differentiate the $\mathrm{HNCO} K_{a}$ ladders, while $K_{a}=1,2$, and 3 exhibited $T_{a} \lesssim 0.6 \mathrm{~K}$. As a general perception in the observed $K_{a}$ ladders, the intensities seem to be in reasonably agreement with the line strengths (i.e. Einstein coefficients) found in spectroscopic databases (Section 2), since they present a slight increment as a function of the frequency. That tendency can also be appreciated in Table 1, where we present the spectroscopic properties and estimated fluxes (i.e. integrated intensities) of the HNCO lines.

Among all the spectral lines, only two are likely affected by blended or contaminant emission.Those are the $11_{0,11}$ $10_{0,10}$, and $13_{0,13}-12_{0,12}$ transitions identified at 241774.032 and $285721.951 \mathrm{MHz}$, respectively (see Fig. 1). For the first one, two neighbour lines of $\mathrm{CH}_{3} \mathrm{OH}$, appearing at $241767.247 \mathrm{MHz}\left(5_{-1,5}\right.$ $\left.4_{-1,4} \mathrm{E}\right)$ and $241791.367 \mathrm{MHz}\left(5_{0,5}-4_{0,4}, \mathrm{~A}\right)$, are likely responsible for the blending. Those transitions were reported in previous works by Loren \& Mundy (1984) in OMC-1. For the second one, it was found partially blended by a broad line likely associated to $\mathrm{SO}_{2} \mathrm{v}=0$ $\left(17_{3,15}-17_{2,16}\right)$ at the frequency $\sim 285743 \mathrm{MHz}$.

Table 1 lists the spectroscopic properties of all the HNCO transitions. The first part of that table presents the $\mathrm{HNCO} K_{a}=0$ spectral characterization. The lowest and highest $K_{a}=0$ transitions are HNCO $8_{0,8}-7_{0,7}$ and $16_{0,16}-15_{0,15}$ identified at the frequencies $\sim 175843.695$ and $351633.257 \mathrm{MHz}$, respectively. Likewise, the upper energy levels $\left(E_{u}\right)$ and Einstein coefficients $\left(A_{u l}\right)$ that govern those transitions are in the ranges $E_{u} \sim 37.98-143.46 \mathrm{~K}$ and $A_{u l}$ $\sim(7.43-61.3) \times 10^{-5} \mathrm{~s}^{-1}$, respectively. Gaussian functions are used to fit parameters as fluxes, full width at half-maximum (FWHM) and $V_{l s r}$ values, all of them in units of $\mathrm{K} \mathrm{km} \mathrm{s}^{-1}, \mathrm{~km} \mathrm{~s}^{-1}$, and $\mathrm{km} \mathrm{s}^{-1}$, respectively. As presented in Table 1, the rest frequencies of the lines are well identified at $V_{l s r} \approx-90 \mathrm{~km} \mathrm{~s}^{-1}$ and the linewidths present a low dispersion of FWHM values, between $\sim 5.5$ and $6.7 \mathrm{~km} \mathrm{~s}^{-1}$. Fluxes are found to be in the range $\sim 3.63-8.02 \mathrm{~K} \mathrm{~km} \mathrm{~s}^{-1}$, with the highest values for the lines at highest frequencies, according to APEX SHeFI 2. For the mentioned range, the lowest and highest flux values correspond to $\mathrm{HNCO} 9_{0,9}-8_{0,8}$ and $15_{0,15}-14_{0,14}$ at 197821.461 and 329664.367 MHz, respectively.

\subsubsection{HNCO $\mathrm{K}_{\mathrm{a}}=1$}

17 lines of $\mathrm{HNCO} K_{a}=1$ have been observed as spectral pairs within the same rotational transitions. Their spectra are exhibited in Fig. 2. As we have already done for $K_{a}=0$ ladder, $K_{a}=1$ transitions have been chosen and superposed over the spectral survey. The intensities and fluxes of the $K_{a}=1$ ladders are lower than those with $K_{a}=0$. The $K_{a}=1$ lines have been observed across a wide frequency interval, from $\sim 175189.027$ to $352897.581 \mathrm{MHz}$, and they are stronger as the frequency increases. As we have highlighted for the $K_{a}=0$ ladder, such tendency can be corroborated through the spectrum and 
Table 1. Lines of HNCO detected in G331. The transitions are presented following the order of the $K_{a}$-ladder numbers, $K_{a}=0, K_{a}=1$, and $K_{a}=2$. The integrated flux $\left(\mathrm{K} \mathrm{km} \mathrm{s}^{-1}\right)$, line width (FWHM, $\left.\mathrm{km} \mathrm{s}^{-1}\right)$, and line position $\left(V_{l s r}, \mathrm{~km} \mathrm{~s}^{-1}\right)$ are estimated from Gaussian fits.

\begin{tabular}{|c|c|c|c|c|c|c|c|}
\hline $\begin{array}{l}\text { Frequency } \\
(\mathrm{MHz})\end{array}$ & $\begin{array}{c}\text { Transition } \\
\left(J_{k_{a}, k_{c}}\right)_{u}-\left(J_{k_{a}, k_{c}}\right)_{l}\end{array}$ & $\begin{array}{c}A_{u l} \\
\left(10^{-5} \mathrm{~s}^{-1}\right)\end{array}$ & $\begin{array}{c}E_{u} \\
(\mathrm{~K})\end{array}$ & $\begin{array}{l}\text { Integrated flux } \\
\left(\mathrm{K} \mathrm{km} \mathrm{s}^{-1}\right)\end{array}$ & $\begin{array}{l}\text { FWHM } \\
\left(\mathrm{km} \mathrm{s}^{-1}\right)\end{array}$ & $\begin{array}{c}V_{l s r} \\
\left(\mathrm{~km} \mathrm{~s}^{-1}\right)\end{array}$ & $\begin{array}{l}\text { NIST } \\
\text { reference }\end{array}$ \\
\hline 175843.695 & $8_{0,8}-7_{0,7}$ & 7.43 & 37.98 & $3.71 \pm 0.03$ & $5.91 \pm 0.05$ & $-90.23 \pm 0.02$ & - \\
\hline 197821.461 & $90,9-80,8$ & 10.70 & 47.47 & $3.63 \pm 0.03$ & $5.86 \pm 0.07$ & $-90.36 \pm 0.03$ & - \\
\hline 219798.274 & $10_{0,10}-9_{0,9}$ & 14.70 & 58.02 & $4.53 \pm 0.05$ & $5.53 \pm 0.07$ & $-90.50 \pm 0.03$ & [1] \\
\hline 241774.032 & $11_{0,11}-10_{0,10}$ & 19.60 & 69.62 & $5.35 \pm 0.33$ & $5.71 \pm 0.44$ & $-90.47 \pm 0.17$ & {$[3]$} \\
\hline 263748.625 & $12_{0,12}-11_{0,11}$ & 25.60 & 82.28 & $4.86 \pm 0.08$ & $5.95 \pm 0.12$ & $-90.66 \pm 0.04$ & {$[1]$} \\
\hline 285721.951 & $13_{0,13}-12_{0,12}$ & 32.60 & 95.99 & $7.90 \pm 0.10$ & $6.70 \pm 0.10$ & $-90.76 \pm 0.04$ & - \\
\hline 307693.905 & $14_{0,14}-13_{0,13}$ & 40.90 & 110.76 & $7.24 \pm 0.03$ & $6.07 \pm 0.03$ & $-90.56 \pm 0.01$ & - \\
\hline 329664.367 & $15_{0,15}-14_{0,14}$ & 50.40 & 126.58 & $8.02 \pm 0.07$ & $6.42 \pm 0.08$ & $-90.64 \pm 0.03$ & - \\
\hline 351633.257 & $16_{0,16}-15_{0,15}$ & 61.30 & 143.46 & $6.79 \pm 0.06$ & $6.60 \pm 0.08$ & $-90.51 \pm 0.03$ & [7] \\
\hline 175189.027 & $8_{1,8}-7_{1,7}$ & 7.16 & 81.11 & $1.01 \pm 0.01$ & $5.08 \pm 0.07$ & $-90.93 \pm 0.03$ & - \\
\hline 176472.191 & $8_{1,7}-7_{1,6}$ & 7.31 & 81.39 & $0.76 \pm 0.01$ & $4.60 \pm 0.10$ & $-90.97 \pm 0.03$ & - \\
\hline 197085.416 & $9_{1,9}-8_{1,8}$ & 10.30 & 90.57 & $0.68 \pm 0.01$ & $4.42 \pm 0.08$ & $-90.99 \pm 0.03$ & - \\
\hline 198528.881 & $9_{1,8}-81,7$ & 10.50 & 90.92 & $1.01 \pm 0.01$ & $5.60 \pm 0.10$ & $-90.85 \pm 0.04$ & - \\
\hline 218981.009 & $10_{1,10}-9_{1,9}$ & 14.20 & 101.08 & $1.75 \pm 0.05$ & $5.73 \pm 0.21$ & $-90.67 \pm 0.07$ & {$[1]$} \\
\hline 220584.751 & $10_{1,9}-9_{1,8}$ & 14.50 & 101.50 & $1.73 \pm 0.06$ & $6.29 \pm 0.31$ & $-90.79 \pm 0.10$ & [2] \\
\hline 240875.727 & $11_{1,11}-10_{1,10}$ & 19.00 & 112.64 & $1.56 \pm 0.03$ & $5.48 \pm 0.12$ & $-90.91 \pm 0.05$ & [3] \\
\hline 242639.704 & $11_{1,10}-10_{1,9}$ & 19.50 & 113.15 & $1.74 \pm 0.04$ & $5.52 \pm 0.13$ & $-90.67 \pm 0.05$ & [3] \\
\hline 262769.477 & $12_{1,12}-11_{1,11}$ & 24.80 & 125.25 & $1.80 \pm 0.10$ & $5.80 \pm 0.50$ & $-90.80 \pm 0.20$ & - \\
\hline 264693.655 & $12_{1,11}-11_{1,10}$ & 25.40 & 125.85 & $1.59 \pm 0.04$ & $5.21 \pm 0.19$ & $-90.85 \pm 0.07$ & [4] \\
\hline 284662.172 & $13_{1,13}-12_{1,12}$ & 31.70 & 138.91 & $2.13 \pm 0.03$ & $5.30 \pm 0.10$ & $-90.86 \pm 0.04$ & - \\
\hline 286746.514 & $13_{1,12}-12_{1,11}$ & 32.40 & 139.61 & $2.53 \pm 0.02$ & $5.53 \pm 0.06$ & $-90.91 \pm 0.02$ & - \\
\hline 306553.733 & $14_{1,14}-13_{1,13}$ & 39.70 & 153.62 & $2.13 \pm 0.02$ & $5.91 \pm 0.07$ & $-90.86 \pm 0.02$ & - \\
\hline 308798.184 & $14_{1,13}-13_{1,12}$ & 40.60 & 154.43 & $2.74 \pm 0.02$ & $5.83 \pm 0.07$ & $-90.86 \pm 0.02$ & - \\
\hline 330848.569 & $15_{1,14}-14_{1,13}$ & 50.10 & 170.31 & $4.64 \pm 0.05$ & $7.40 \pm 0.10$ & $-90.56 \pm 0.04$ & [5] \\
\hline 350333.059 & $16_{1,16}-15_{1,15}$ & 59.70 & 186.20 & $3.24 \pm 0.04$ & $5.84 \pm 0.09$ & $-90.81 \pm 0.03$ & [6] \\
\hline 352897.581 & $16_{1,15}-15_{1,14}$ & 61.00 & 187.25 & $2.55 \pm 0.03$ & $5.25 \pm 0.08$ & $-90.81 \pm 0.03$ & - \\
\hline 175791.267 & $8_{2,7}-7_{2,6}$ & 6.65 & 208.25 & $0.37 \pm 0.01$ & $7.60 \pm 0.30$ & $-92.20 \pm 0.10$ & - \\
\hline 175792.957 & $8_{2,6}-7_{2,5}$ & 6.65 & 208.25 & $0.41 \pm 0.03$ & $7.90 \pm 0.60$ & $-89.40 \pm 0.30$ & - \\
\hline 197762.939 & $9_{2,8}-8_{2,7}$ & 9.66 & 217.74 & $0.51 \pm 0.02$ & $9.10 \pm 0.40$ & $-92.50 \pm 0.20$ & - \\
\hline 197765.372 & $9_{2,7}-8_{2,6}$ & 9.66 & 217.74 & $0.52 \pm 0.05$ & $9.20 \pm 0.40$ & $-88.8 \pm 0.10$ & - \\
\hline $219733.850^{\dagger}$ & $10_{2,9}-9_{2,8}$ & 13.50 & 228.29 & - & - & - & [3] \\
\hline $219737.193^{\dagger}$ & $10_{2,8}-9_{2,7}$ & 13.50 & 228.29 & - & - & - & [3] \\
\hline $241703.852^{a}$ & $11_{2,10}-10_{2,9}$ & 18.10 & 239.89 & - & - & - & - \\
\hline 241708.312 & $11_{2,9}-10_{2,8}$ & 18.10 & 239.89 & $0.67 \pm 0.04$ & $4.90 \pm 0.40$ & $-91.20 \pm 0.10$ & - \\
\hline 285640.923 & $13_{2,12}-12_{2,11}$ & 30.40 & 266.25 & $0.63 \pm 0.05$ & $6.40 \pm 0.60$ & $-91.60 \pm 0.20$ & - \\
\hline 285648.301 & $13_{2,11}-12_{2,10}$ & 30.40 & 266.25 & $0.53 \pm 0.04$ & $6.20 \pm 0.60$ & $-91.30 \pm 0.1$ & - \\
\hline 307607.799 & $14_{2,13}-13_{2,12}$ & 38.20 & 281.01 & $0.64 \pm 0.02$ & $7.70 \pm 0.40$ & $-91.70 \pm 0.1$ & - \\
\hline 307617.020 & $14_{2,12}-13_{2,11}$ & 38.20 & 281.01 & $0.47 \pm 0.02$ & $6.10 \pm 0.30$ & $-90.70 \pm 0.10$ & - \\
\hline 329573.452 & $15_{2,14}-14_{2,13}$ & 47.20 & 296.83 & $0.57 \pm 0.05$ & $5.50 \pm 0.60$ & $-91.30 \pm 0.20$ & - \\
\hline 329584.800 & $15_{2,13}-14_{2,12}$ & 47.20 & 296.83 & $0.64 \pm 0.08$ & $7.00 \pm 1.00$ & $-90.8 \pm 0.30$ & - \\
\hline 351537.795 & $16_{2,15}-15_{2,14}$ & 57.50 & 313.70 & $0.72 \pm 0.07$ & $5.80 \pm 0.70$ & $-91.60 \pm 0.20$ & - \\
\hline 351551.573 & $16_{2,14}-15_{2,13}$ & 57.50 & 313.70 & $0.46 \pm 0.04$ & $5.70 \pm 0.70$ & $-90.80 \pm 0.20$ & - \\
\hline
\end{tabular}

Notes and labels: Columns 5-7 list Gaussian fit parameters with their respective uncertainties. ${ }^{\dagger}$ Weak and unresolved lines. ${ }^{a}$ Line likely affected by dominant emission of $\mathrm{CH}_{3} \mathrm{OH}$. NIST references, when available: [1] Armstrong \& Loren (1984); [2] Loren \& Mundy (1984); [3] Sutton et al. (1985); [4] Greaves \& White (1991); [5] Sutton et al. (1991); [6] MacDonald et al. (1996); [7] Jewell et al. (1989).

the fitted parameters listed in the middle part of Table 1. Concerning to problems affecting the spectral emission, almost all the lines are clean or free of contamination.

Two aspects can be highlighted from the spectra exhibited in Fig. 2. On the one hand, the spectral profiles are not totally symmetrical, exhibiting a tail extended towards low-frequency values, in some cases. On the other hand, regarding contamination or problems in the spectra, the HNCO $10_{1,9}-9_{1,8}$ line at $220584.751 \mathrm{MHz}$ presents a neighbour unidentified emission that could be $\mathrm{CH}_{3} \mathrm{CN} 12{ }_{6}-11_{6}$ at $220594.431 \mathrm{MHz}$, which was previously reported in OMC-1 (Loren \& Mundy 1984). Moreover, in the panel of the HNCO 12 $1,12-$ $11_{1,11}$ line at $262769.477 \mathrm{MHz}$, a feature in absorption appears at $\sim 262787 \mathrm{MHz}$ affecting the whole spectrum. Such absorption is likely associated with water vapour as it has been discussed in studies about the atmospheric transparency at Chajnantor. ${ }^{10}$

Concerning the line profiles, the spectral tail mentioned above has also been evidenced in $\mathrm{HNCO}$ lines detected in $\mathrm{OH} 231.8+4.2$, which is an oxygen-rich circumstellar envelope around an intermediatemass evolved star also harbouring a bipolar molecular outflow (Velilla Prieto et al. 2015). So we speculate that such asymmetry in the lines could be due to outflow activity. In addition, it is also worth mentioning that such tail is more notorious in some transitions than others. It also seems to be better traced by the ladder $K_{a}=1$

\footnotetext{
${ }^{10} \mathrm{https}: / /$ almascience.eso.org/about-alma/atmosphere-model
} 

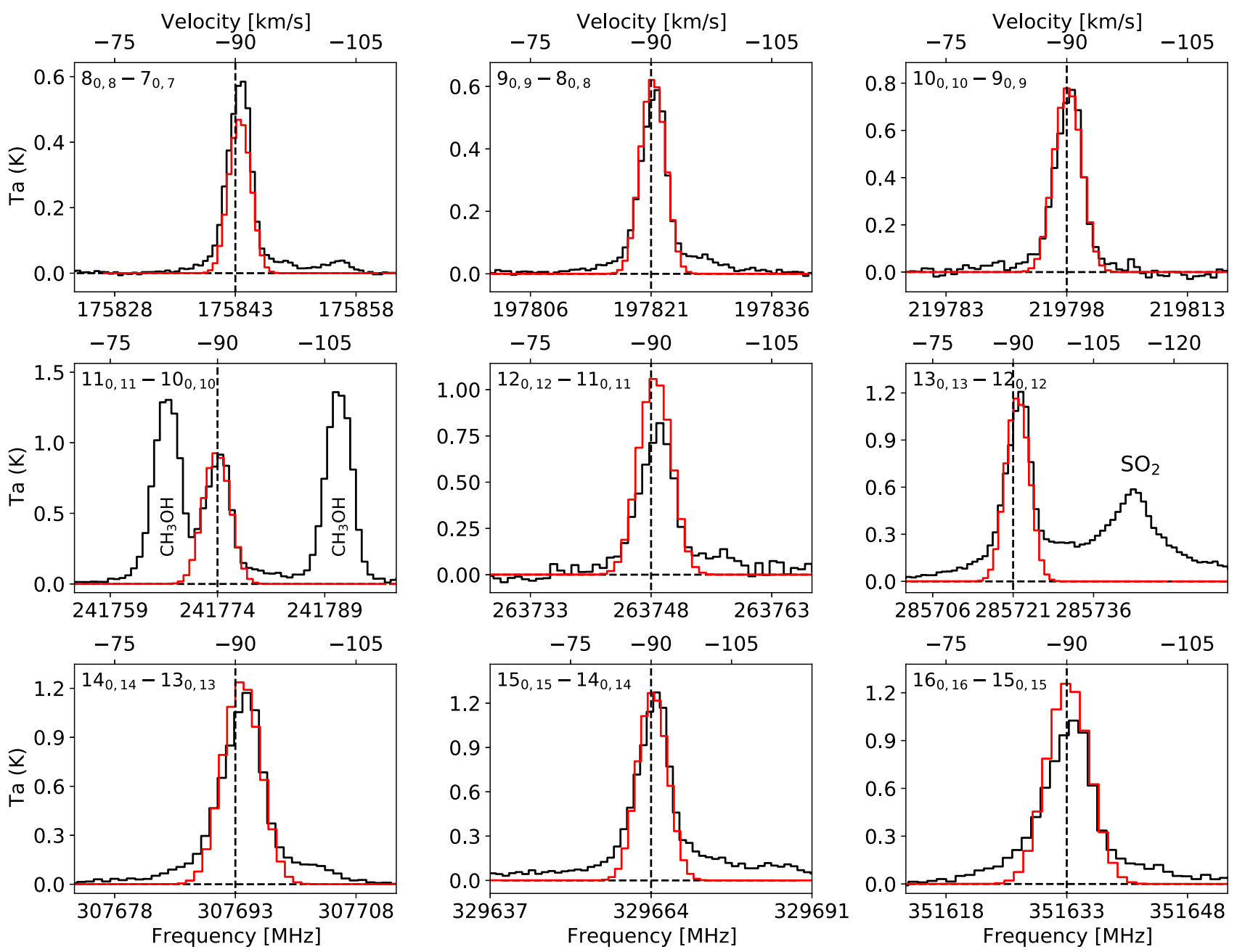

Figure 1. HNCO emission lines $\left(K_{a}=0\right)$ and their LTE models, exhibited as black and red histograms, respectively. The line intensities, $y$-axes, are given in units of antenna temperature, the $x$-axes represent the frequency (bottom) and velocity (top). The horizontal and vertical dashed lines represent the baseline and the rest velocity of G331, respectively. The transitions are indicated in the top left corner of each plot. The spectral resolution was smoothed to $1 \mathrm{~km} \mathrm{~s}^{-1}$.

than $K_{a}=0$. In Fig. 3, we emphasize the observed tails in the HNCO $8_{1,8}-7_{1,7}$ and $15_{1,14}-14_{1,13}$ lines, detected at 175189.027 and $330848.569 \mathrm{MHz}$, respectively, since both lines exhibit remarkably well the one-side tail extended up to $-70 \mathrm{~km} \mathrm{~s}^{-1}$. Comparing both lines, we note that the second one is more intense and stronger by a factor $\sim 5$ if we take, for instance, the ratio from their fluxes. Although other studies found spectral profiles of HNCO (Velilla Prieto et al. 2015) similar to those observed here, we took into account a scenario of possible contamination affecting the HNCO emission (Fig. 3). However, such scenario is unlikely due to the rare species and/or transitions theoretically predicted. In the case of the HNCO $8_{1,8^{-}}$ $7_{1,7}$ line detected at $175189.027 \mathrm{MHz}$, transitions of e.g. $\mathrm{CH}_{2} \mathrm{ND}$, $\mathrm{C}_{2} \mathrm{H}_{5} \mathrm{OH}$, and $\mathrm{i}-\mathrm{C}_{3} \mathrm{H}_{7} \mathrm{CN}$ are predicted at $\sim 175185.19,175$ 188.99, and $175189.44 \mathrm{MHz}$. Regarding the HNCO 151,14-14,13 line detected at $330848.569 \mathrm{MHz}$, which was catalogued in Sgr B2(N) by Sutton et al. (1991), transitions of e.g. HCOCN and ${ }^{15} \mathrm{NH}_{2} \mathrm{HCO}$ at $\sim 330845.85$ and $330851.20 \mathrm{MHz}$, respectively, could be possible contaminants. Thus, the observed spectral profiles of HNCO seem to be much better explained by the outflow activity of G331.

In spite of the spectral asymmetries shown in Fig. 3, a relative percentage was estimated by considering the area under the Gaussian fits with respect to the whole area integrated within $-70 \leq$
$V_{l s r}\left(\mathrm{~km} \mathrm{~s}^{-1}\right) \leq-110$. Thus, the areas under the Gaussian fits represent up to $\approx 80$ per cent of the total area under the spectra. Although these lines have different intensities and fluxes (Fig. 3b), the percentages are similar as both lines have similar spectral tails. Velilla Prieto et al. (2015) also observed this wing, through spectral lines of HNCO, but in outflows of AGB stars.

\subsubsection{HNCO $\mathrm{K}_{\mathrm{a}}=2$}

16 transitions of HNCO $K_{a}=2$ have been identified across the survey, they appear as spectral pairs partially resolved. The spectral lines are exhibited in Fig. 4, where it can be noted that their emission are noisier and weaker than the previously analysed $K_{a}$ ladders; those were aspects that put constraints on the line identification of the HNCO $K_{a}=2$ transitions. Likewise, it can be observed that the intensity of the lines increases with the frequency. In addition, the spectral pairs are more spaced as the frequency increases. For instance, by comparing the two panels centred at 175792 and $329579 \mathrm{MHz}$ of Fig. 4, it can be noted that the spectral pair of the second one, $15_{2,14}-14_{2,13}$ and $15_{2,13}-14_{2,12}$, is better resolved than the first one, $8_{2,7}-7_{2,6}$ and $8_{2,6}-7_{2,5}$. Apart from the HNCO blending mentioned above, only the $K_{a}=2$ transitions at $\sim 241706 \mathrm{MHz}$ are 

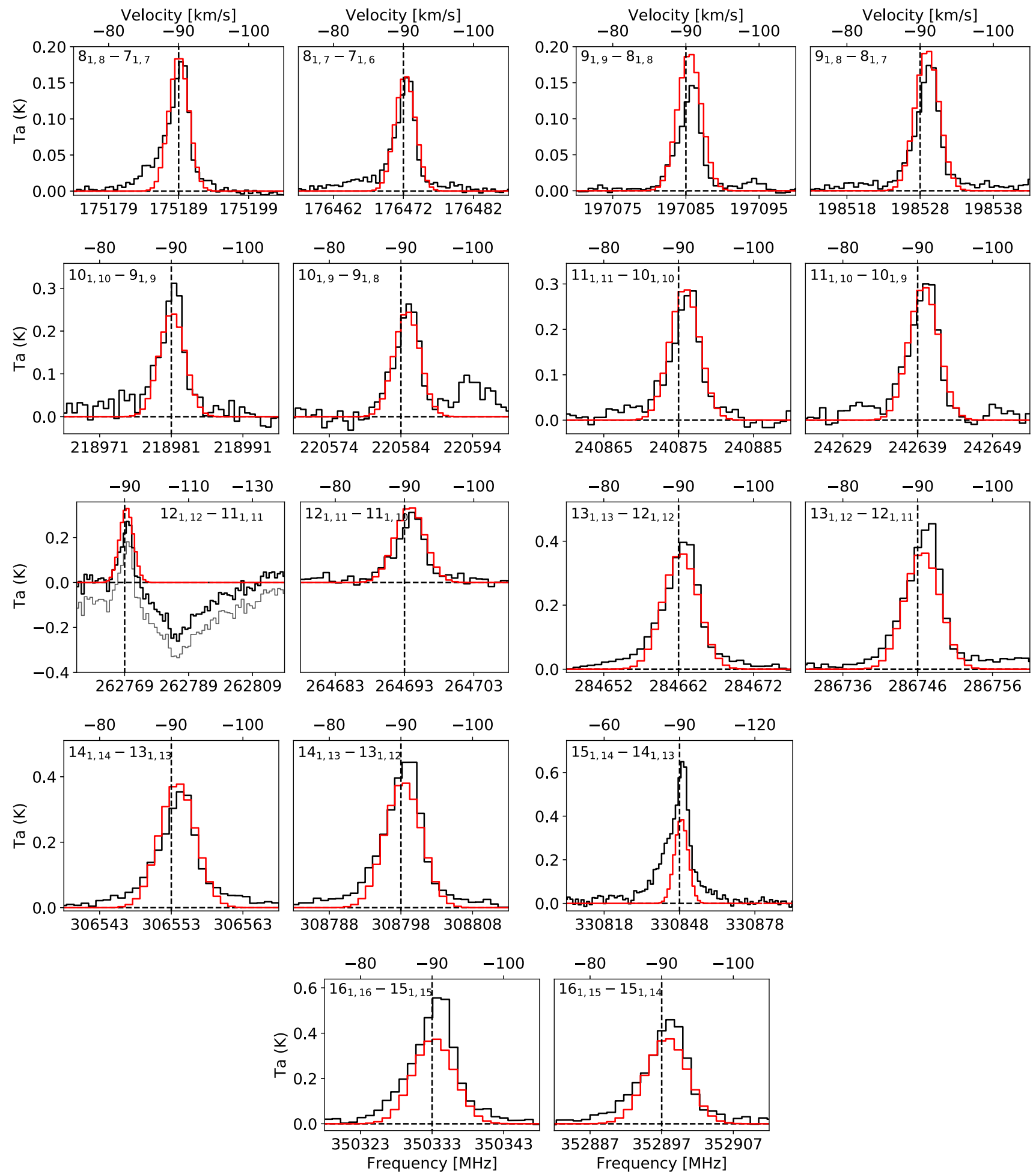

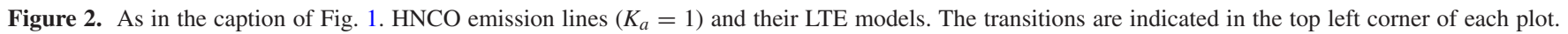

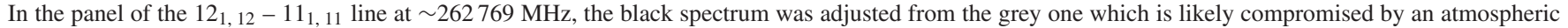
issue at $\sim 262785 \mathrm{MHz}$ (see the text).

affected by a neighbour and intense line of $\mathrm{CH}_{3} \mathrm{OH}\left(5_{0,5}-4_{0,4}, \mathrm{E}\right)$ identified at $\sim 241700 \mathrm{MHz}$ (see Fig. 4). Concerning the line profiles, the lines do not exhibit the spectral tails like those highly evidenced in the HNCO $K_{a}=1$ ladder.

In the lowest part of Table 1, we present the parameters of the observed HNCO $K_{a}=2$ transitions. Summarizing, the lowest and highest transitions are identified at the rest frequencies 175791.267 and $351551.573 \mathrm{MHz}$, respectively. Their upper energy levels, which are in the range $E_{u} \sim 208.25-313.70 \mathrm{~K}$, are the highest in comparison with the other $K_{a}$ ladders. Concerning the Einstein coefficients, they are in the range $A_{u l} \sim(6.65-57.5) \times 10^{-5} \mathrm{~s}^{-1}$, similar to those of the other $K_{a}$ ladders. In order to quantify the spectral emission, Gaussian 


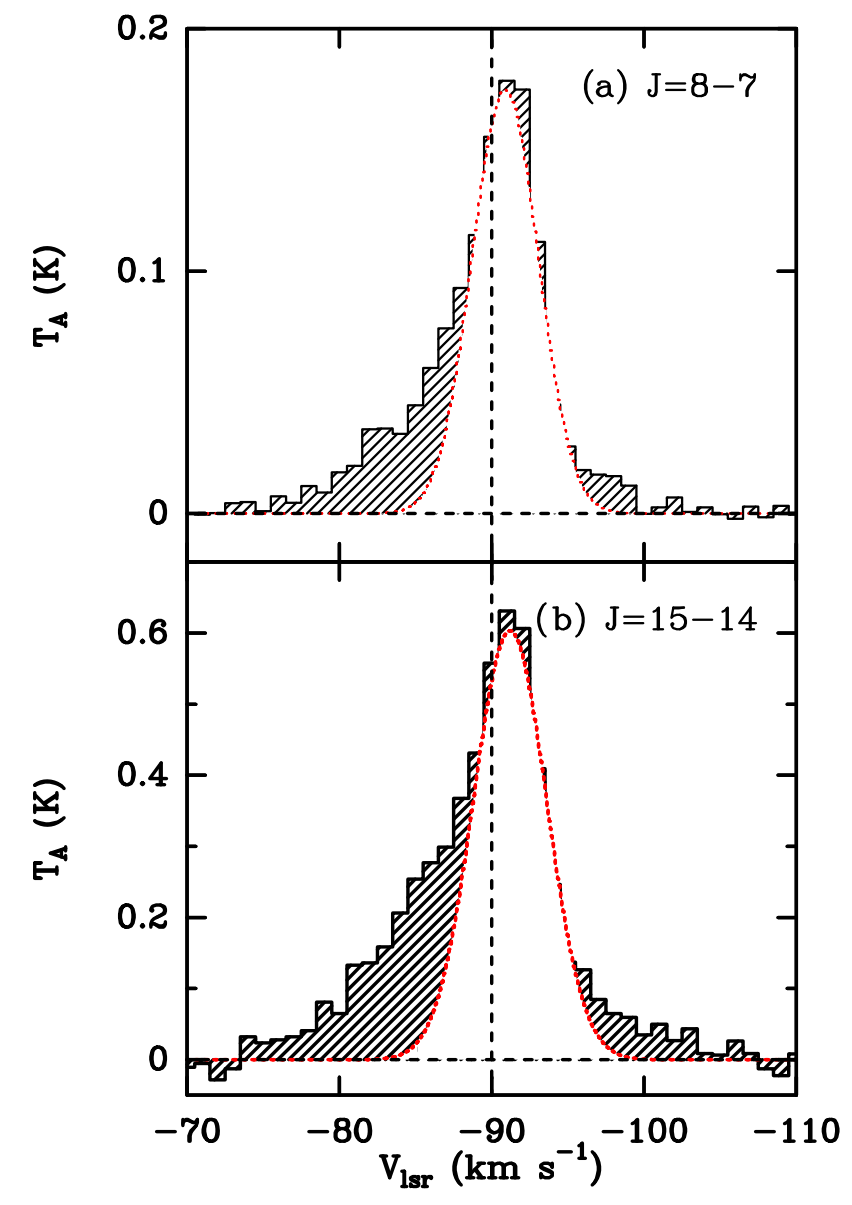

Figure 3. Spectral wings observed in the (a) HNCO $8_{1,8}-7_{1,7}$ and (b)

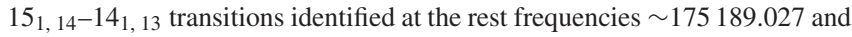
$330848.569 \mathrm{MHz}$, respectively. The diagonal patterns depict the area under the spectra from -70 to $-110 \mathrm{~km} \mathrm{~s}^{-1}$. The red dotted lines depict the areas under the Gaussian fits.

functions have been adjusted to estimate the line parameters, whose results are presented in Table 1 . Since the lines are partially blended, the estimation of the Gaussian fits has been difficult, mainly for the lowest frequency transition pairs observed at $\sim 175792,197764$, 219735, and $241706 \mathrm{MHz}$. In addition to that problem, and due to the weakness of the emission, the resultant Gaussian fits are not as good as those for the previously analysed $K_{a}$ ladders; as a consequence, the LTE analysis of the HNCO $K_{a}=2$ lines, e.g. the match between models and observations (Fig. 4), might not be as reliable as those obtained from the strongest $K$ ladders observed in this work (Figs 1 and 2). Regarding the rest frequencies, they were found more dispersed with respect to the systemic velocity, approximately between -88 and $-92 \mathrm{~km} \mathrm{~s}^{-1}$. Intensities and fluxes were found to be as the weakest among all the $K_{a}$ ladders, see Table 1 . The transitions $10_{2,9}-9_{2,8}, 10_{2,8}-9_{2,7}$, and $11_{2,10}-10_{2,9}$ were unable to obtain reliable Gaussian fits.

\subsubsection{HNCO $\mathrm{K}_{\mathrm{a}}=3$}

A subtle evidence for a HNCO $K_{a}=3$ line has been found. The HNCO $13_{3,10}-12_{3,9}$ transition is identified at the rest frequency $285541.575 \mathrm{MHz}$, and is exhibited in Fig. 5. As expected, the spectral line is weak and has the highest upper energy level identified in this work, $E_{u}=470.91 \mathrm{~K}$, with $A_{u l}=2.78 \times 10^{-4} \mathrm{~s}^{-1}$. As a consequence, such emission would suggest a higher excitation condition than that found from the other $\mathrm{HNCO} K_{a}=0,1$, and 2 ladders. As can be noted by the models at two different excitation temperatures displayed in Fig. 5, the highest temperature does reproduce better the line profile. Analysing other species for the peak, $\mathrm{CH}_{3} \mathrm{OCN}$ at $285539.098 \mathrm{MHz}$, DNCO at $285539.531 \mathrm{MHz}$, or acetone $\left(\mathrm{CH}_{3}\right)_{2} \mathrm{CO}$ at $285542.349 \mathrm{MHz}$ could be candidate transitions. The predicted position in the spectrum for those species are also indicated in Fig. 5.

\subsection{LTE analysis}

In order to estimate the physical conditions traced by HNCO in G331, we constructed rotational diagrams. They are an important method to infer source properties from molecular line emissions (see Goldsmith \& Langer 1999, for a complete description and discussion). They basically consist of a plot of the column density per statistical weight, for a given number of molecular rotational energy levels, as a function of their energies above the ground state. More specifically, if LTE is assumed for the source, the level populations can be represented by the Boltzmann distribution and the rotational diagram is described by equation (1). Moreover, if the emission lines can also be considered as optically thin, the plotted function is a straight line with a slope defined by $1 / T_{e x}$ from which is possible to derive the excitation temperature of the source where it is the molecule, which should be equal to the kinetic temperature of the gas in LTE conditions. Thus,

$\ln \left(\frac{N_{u}}{g_{u}}\right)=\ln \left(\frac{3 k W}{8 \pi^{3} v S_{u l} \mu^{2} g_{u}}\right)=\ln \left(\frac{N}{Q}\right)-\frac{E_{u}}{k T_{e x}}$

where $N_{u}$ is the column density of the upper level, $g_{u}$ the degeneracy of the upper level, $k$ is the Boltzmann constant, $W$ the integrated intensity of the transition, $v$ the rest frequency of the line, $S_{u l}$ is the line strength of the transition, $\mu$ is the dipolar moment, $N$ is the total column density, $Q$ is the internal partition function of the molecule, and $E_{u}$ the upper level energy of the transition.

For the construction of the rotational diagram, it is also considered a beam dilution factor associated with a point-like emitter region. The correction can be performed by the addition of the term $\ln \left(\Delta \Omega_{a} / \Delta \Omega_{s}\right)$ on the right-hand side of equation (1; Goldsmith \& Langer 1999), where $\Delta \Omega_{a}$ is the antenna solid angle and $\Delta \Omega_{s}$ is the source solid angle. This ratio correlates the subtended angle of the source with the solid angle of the antenna beam. For the HNCO emission, observed through several spectral lines, we adopt a hypothetical source size of 5 arcsec to evaluate the beam dilution effects, although, under the hypothesis that the emission fills the antenna beam, a rotational diagram solution was also constructed considering 30 arcsec. The LTE treatment of HNCO was motivated by radiative analyses of $\mathrm{HC}_{3} \mathrm{~N}$ in $\mathrm{G} 331$, although the physicochemical origin of both molecules is different. For instance, in previous works, various lines of $\mathrm{HC}_{3} \mathrm{~N}$, including its ${ }^{13} \mathrm{C}$ isotopologues, were analysed with APEX (Duronea et al. 2019), as well as mapped with $A L M A$ via the $\mathrm{HC}_{3} \mathrm{~N} J=38-37$ transition (Hervías-Caimapo et al. 2019). Furthermore, in the case of optically thick transitions, the optical depth correction can be performed by the correction factor $C_{\tau}$ (equation 3) as shown in equation (2; as derived in Goldsmith \& Langer 1999):

$N_{u, \text { thick }}=N_{u, \text { thin }} \times C_{\tau}$

$C_{\tau}=\frac{\tau}{1-e^{-\tau}}$,

where $\tau$ is the optical depth.

The rotational diagrams with and without opacity correction (2) are presented in Fig. 6. Regarding the rotational diagram corrected 

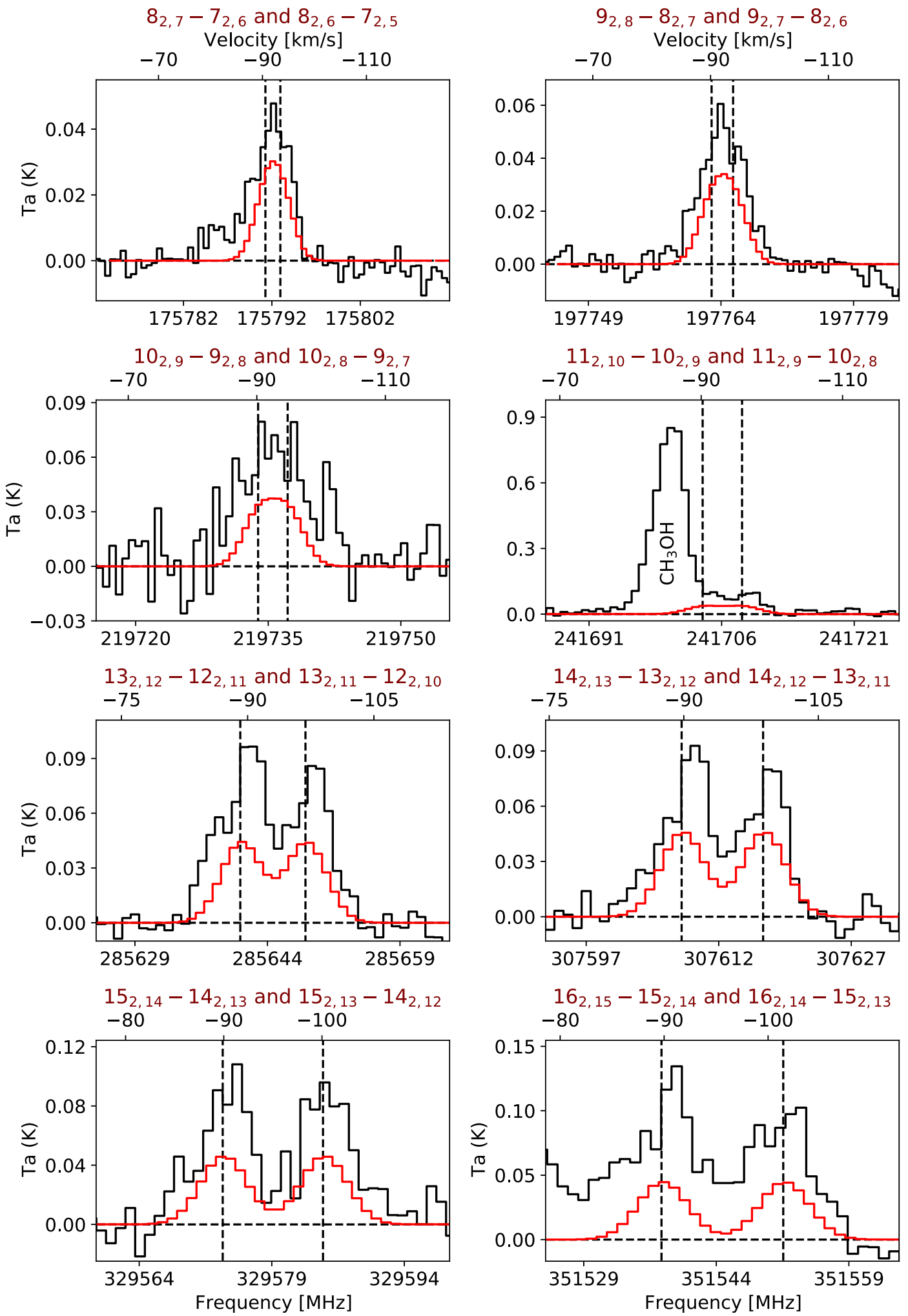

Figure 4. As in the caption of Fig. 1. HNCO emission lines $\left(K_{a}=2\right)$ and their LTE models. The transitions are indicated in the top left corner of each plot.

by the opacity, it considers only the non-blended transitions of HNCO because the opacity correction does not converge on blended transitions, like various of those belonging to the $K_{a}=2$ ladder and identified at lowest frequency values (e.g. the first panels of Fig. 4). The best linear fit for the diagrams gave $N(\mathrm{HNCO})=(3.1 \pm 0.4) \times$
$10^{15} \mathrm{~cm}^{-2}$ and $T_{e x}=59.4 \pm 2.3 \mathrm{~K}$, without the opacity correction; and $N(\mathrm{HNCO})=(3.7 \pm 0.5) \times 10^{15} \mathrm{~cm}^{-2}$ and $T_{e x}=58.8 \pm 2.7 \mathrm{~K}$, by applying the opacity correction. The reduced $\chi^{2}$ values for each fit are 1.12 and 1.21, respectively. Assuming that the HNCO emission is extended, optically thin, and that fills approximately the antenna 


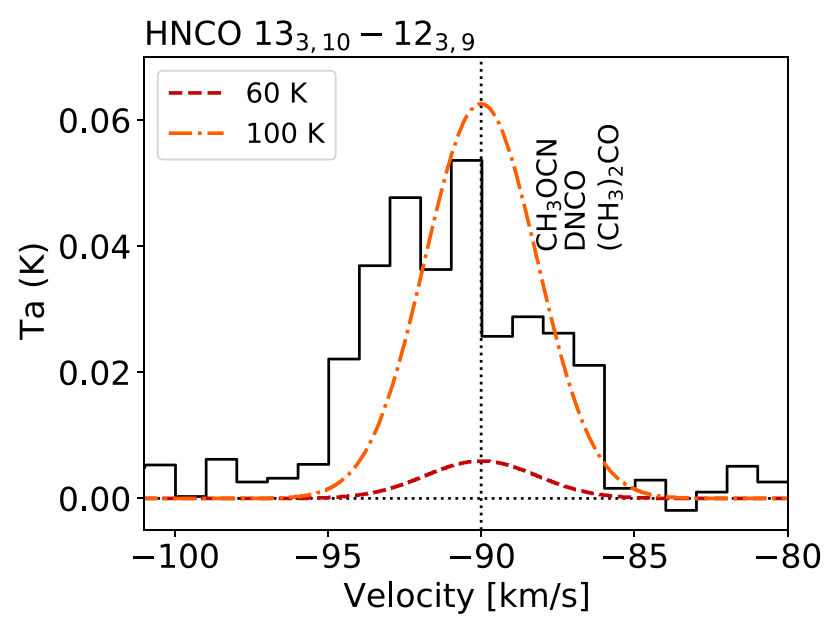

Figure 5. Tentative detection of the HNCO line $\left(13_{3,10}-12_{3,9}\right)$ at the rest frequency $285541.575 \mathrm{MHz}$. The baseline and the source's velocity are represented by the dotted horizontal and vertical lines, respectively. The LTE models at 60 and $100 \mathrm{~K}$, displayed as red dashed and orange dot-dashed lines, respectively, are to illustrate the rotational diagram result and a hypothetical higher excitation condition, respectively. There is a fainter emission next to the HNCO line where we indicated other candidate molecules.
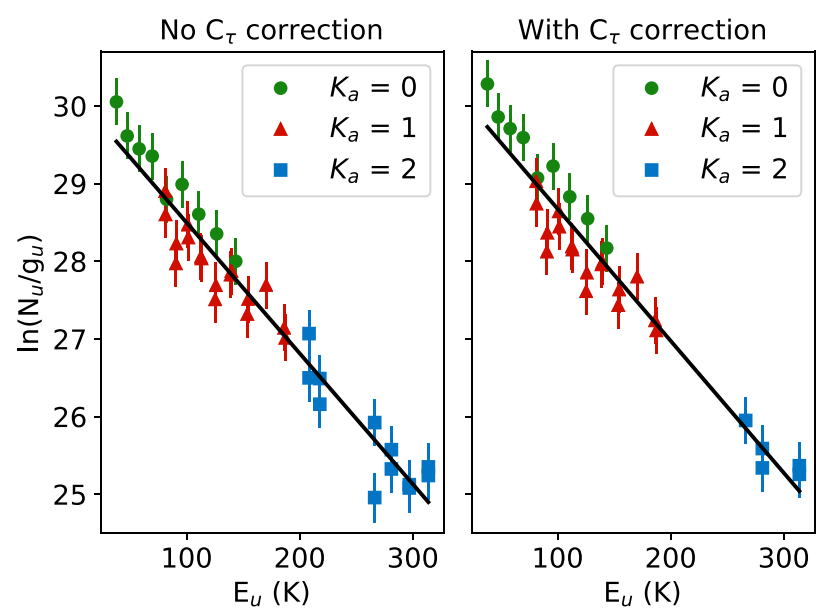

Figure 6. Rotational diagrams of HNCO constructed applying opacity correction (right-hand panel) and without opacity correction (left-hand panel). In both cases, a source size of 5 arcsec was adopted. In second plot, only the lines with no blended/contaminant emission are considered. The best linear fit for each display yields, respectively, $T_{e x}=59.4 \pm 2.3 \mathrm{~K}$ and $N=(3.1 \pm 0.4)$ $\times 10^{15} \mathrm{~cm}^{-2}\left(\chi_{\text {red }}^{2}=1.12\right)$; and $T_{e x}=58.8 \pm 2.7 \mathrm{~K}$ and $N=(3.7 \pm 0.5) \times$ $10^{15} \mathrm{~cm}^{-2}\left(\chi_{\text {red }}^{2}=1.21\right)$. The set of data with different $K_{a}$ values are also represented in the plots.

beam, we evaluated a rotational diagram adopting $30 \mathrm{arcsec}$, the best linear fit $\left(\chi_{\text {red }}^{2}=1.98\right)$ gave $N(\mathrm{HNCO})=(1.4 \pm 0.2) \times 10^{14} \mathrm{~cm}^{-2}$ and $T_{e x}=65.5 \pm 3.7 \mathrm{~K}$. With similar results, such temperatures around $60 \mathrm{~K}$ might indicate that HNCO molecules are concentrated in external and colder regions of the core, according to the proposed shell-like structure of G331 (Merello et al. 2013b; Duronea et al. 2019; Hervías-Caimapo et al. 2019).

Moreover, Churchwell et al. (1986) indicated that HNCO transitions with $E_{u}>40 \mathrm{~K}$ require an excitation temperature of $\sim 70 \mathrm{~K}$ and also that radiative processes are responsible for the excitation of HNCO in Sgr B2, as already mentioned. This suggests that HNCO can be considered as a good probe of the far-infrared radiation field but not of gas properties such as density and kinetic temperature. The only common transition with our work is $10_{0,10}-9_{0,9}$, the others are at lower frequencies (Churchwell et al. 1986). In this sense, according to the similar excitation temperature of $\sim 60 \mathrm{~K}$ obtained with the rotational diagrams, the same trend appears to be maintained at higher HNCO transition levels. This may imply that radiative processes, rather than collisional mechanisms, could also dominate the excitation of HNCO molecules in G331. In this article, it is reported for first time a multiline analysis of $\mathrm{HNCO}$, covering transitions within the energy interval $E_{u} \approx 37-314 \mathrm{~K}$, towards a massive hot molecular core/outflow like G331. Recently, He et al. (2021) studied the spatial distribution of $\mathrm{HNCO} 4_{0,4}-4_{0,3}, \mathrm{SiO} 2$ 1 , and $\mathrm{HC}_{3} \mathrm{~N} 10-9$ in a sample of southern massive star-forming regions, discussing correlations between the morphology of the gas and dust emission. In an earlier work, Martín et al. (2008) proposed how HNCO and CS could be useful molecules to study the influence of shocks and/or the radiation field in nuclear regions of galaxies. Thus, there are follow-up questions that warrant further studies to have a deeper understanding on the role that the outflow activity, the physical conditions of the dust, and collisional mechanisms can play into the formation, distribution, and excitation of HNCO gas in massive protostellar objects like G331.

\section{DISCUSSION}

\subsection{Abundances: comparison with other sources}

From the column densities obtained with the rotational diagram, assuming a source size of 5 arcsec, it is possible to estimate the abundance of $\mathrm{HNCO}$ if the column density of $\mathrm{H}_{2}$ is also known. The most recent values of $N\left(\mathrm{H}_{2}\right)$ derived for $\mathrm{G} 331$ are $9.7 \times 10^{23} \mathrm{~cm}^{-2}$, obtained from the $\mathrm{H}^{13} \mathrm{CO}^{+} / \mathrm{H}_{2}$ ratio of Orion $\mathrm{KL}$, and $2.7 \times 10^{23} \mathrm{~cm}^{-2}$, estimated by the superficial density from the continuum emission at $1.2 \mathrm{~mm}$ in G331 (Duronea et al. 2019, and references therein). Therefore, by taking the column density of HNCO corrected by the optical depth, $N(\mathrm{HNCO})=(3.7 \pm 0.5) \times$ $10^{15} \mathrm{~cm}^{-2}$, and adopting $\mathrm{H}_{2}$ column densities values of $2.7 \times 10^{23}$ and $9.7 \times 10^{23} \mathrm{~cm}^{-2}$, the relative abundances of $\mathrm{HNCO}$ are estimated to range from $(3.8 \pm 0.5) \times 10^{-9}$ to $(1.4 \pm 0.2) \times 10^{-8}$.

In Fig. 7, the histograms of the column densities and HNCO abundances for several sources are displayed in order to compare with those derived for G331. The objects shown are high-mass young stellar objects (YSOs) in hot core stages (AFGL 2591, G24.78, G75.78, NGC 6334 IRS1, NGC 7538 IRS1, W3(H2O), and W 33A; Bisschop et al. 2007); low-mass protostar IRAS 16293-2422, in the compact source B (Martín-Doménech et al. 2017) and in the hot corino region (Hernández-Gómez et al. 2019); oxygen-rich circumstellar envelope around an intermediate-mass evolved star $(\mathrm{OH} 231.8+4.2$; Velilla Prieto et al. 2015); and hot molecular core (G10.47+0.03; Gorai et al. 2020).

Concerning the HNCO column densities, G331 and most of the YSOs of Bisschop et al. (2007) present similar values. This might be due to similar evolutionary stage of these objects, with protostars embedded in hot molecular cores. The two sources of IRAS 16293 2422 have higher column densities, which could be connected to the innermost and densest regions of this object (e.g. hot corino). In colder envelopes, the HNCO column densities could be up to four orders of magnitude lower (Hernández-Gómez et al. 2019). The histogram of the abundances, on the other hand, reveals a greater variation among the objects. The HNCO abundances of G75.78 and G331 $1_{M I N}$ are very similar, while G331 $1_{M A X}$ is in better agreement with the other YSOs. 

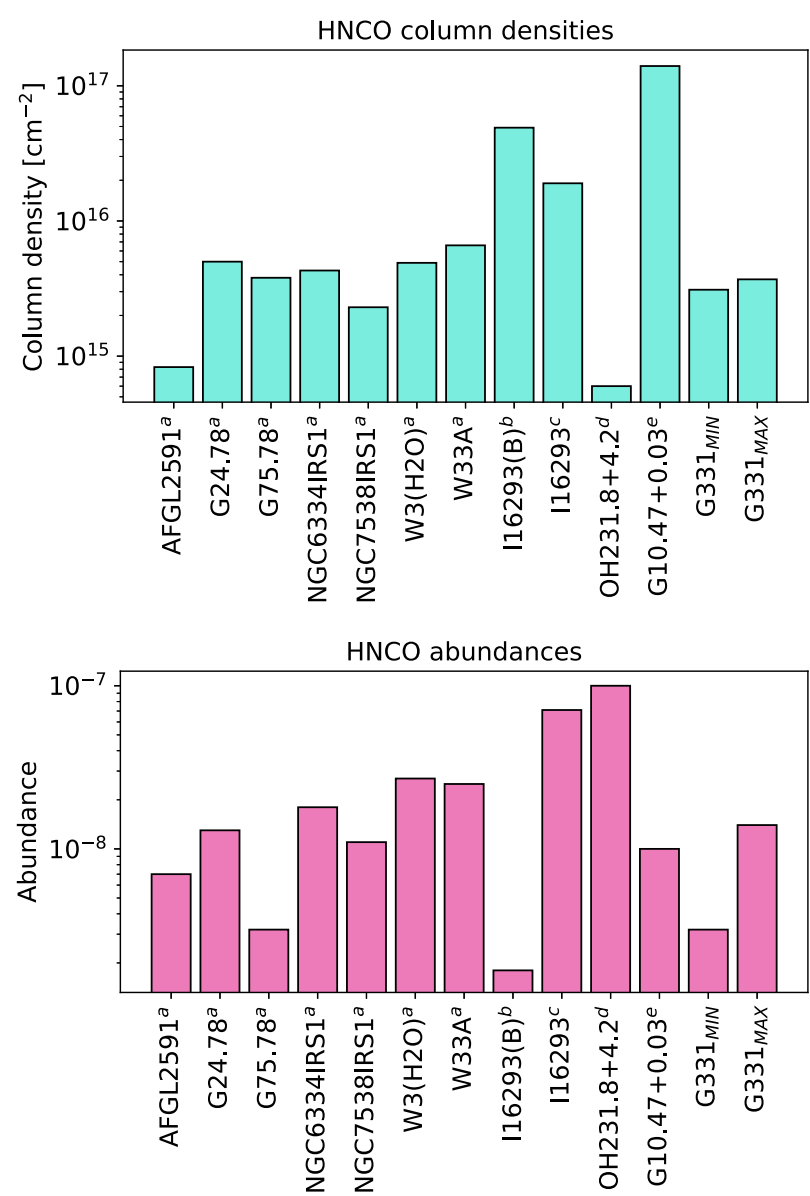

Figure 7. Histograms of the column densities of HNCO (top) and their relative abundances to $\mathrm{H}_{2}$ (bottom) in several sources: ${ }^{a}$ Bisschop et al. (2007), ${ }^{b}$ IRAS 16293-2422 (B, Martín-Doménech et al. 2017), ${ }^{c}$ IRAS 16293-2422 (hot corino, Hernández-Gómez et al. 2019), ${ }^{d}$ Velilla Prieto et al. (2015), ${ }^{e}$ Gorai et al. (2020). The minimum and maximum values (G331 $1_{M I N}$ and G331 $1_{\text {MAX }}$ ) obtained for G331 are also shown.

It is worth noting that the column density reported for G10.47+0.03 is much higher than G331, however the abundance of HNCO in this source is in the range calculated for G331. The most evolved object in this sample, $\mathrm{OH} 231.8+4.2$, shows the lowest column density and the highest abundance of HNCO. Despite these peculiar cases, the values of HNCO column density and abundance in G331 are in agreement with those obtained in most of the hot molecular cores in the histograms.

\subsection{The CHNO isomers: HNCO, HOCN, HCNO, and HONC}

HNCO has three meta-stable isomers of which HCNO (fulminic acid) and HOCN (cyanic acid) as well as HNCO have been observed in the ISM. Isofulminic acid (HONC) is the only meta-stable isomer that has not been detected in the ISM yet. All these isomers are quasi-linear molecules except isofulminic acid, which has a bent structure (Mladenovic \& Lewerenz 2008; Mladenovic et al. 2009). $\mathrm{HCNO}$ and HOCN are much less abundant than HNCO and their occurrence in space has been restricted to a few sources. On the one hand, HCNO was discovered in dark clouds by Marcelino et al. (2009); using observations at the $3 \mathrm{~mm}$ band of the IRAM-30 m telescope, they detected HCNO in the starless cores B1, L1544, L183 as well as in the low-mass star-forming region L1527. On the other hand, HOCN was observed towards the Galactic centre by Brünken et al. (2010); similarly, using observations at the $3 \mathrm{~mm}$ band of IRAM$30 \mathrm{~m}$, they detected transitions of HOCN in a quiescent molecular cloud and in several positions of the Sgr B2 complex.

The dipole moments of the four isomers have been reported and discussed in the literature. The values for HNCO, HCNO, HOCN, and HONC have been estimated to be $\sim 2.08,3.1,3.7$, and $3.13 \mathrm{D}$, respectively (e.g. Hocking et al. 1974; Hocking et al. 1975; Takashi, Tanaka \& Tanaka 1989; Mladenovic \& Lewerenz 2008; Mladenovic et al. 2009). Among the four species, HNCO is the most stable one (by $1.1 \mathrm{eV}$ ), this has been demonstrated through studies about the equilibrium structure and energetic of the CHNO isomers (e.g. Mladenovic \& Lewerenz 2008; Mladenovic et al. 2009). Regarding the abundance ratios between the isomers, Marcelino et al. (2009) determined $\mathrm{HNCO} / \mathrm{HCNO}$ between 40 and 70 in dark clouds and starless cores; Brünken et al. (2010) determined values of $\sim 0.3-$ 0.8 per cent for $\mathrm{HOCN}$ relative to $\mathrm{HNCO}$ towards the Galactic centre.

In this work, HNCO was clearly detected through several spectral lines, as presented in Table 1. The search for HCNO and HOCN, on the other hand, did not yield conclusive results to confirm their presence in G331. About HOCN, the number of predicted transitions with $E_{u}<450 \mathrm{~K}$ is about 60 ; however, only spectral noise has been observed where most of the lines should appear. Two transitions might be speculatively inspected since they are dominated by spectral emission relatively well known in the literature. That is the case of the transition HOCN $9_{3,6}-8_{3,5}$ at the rest frequency $188655.358 \mathrm{MHz}$, which is likely affected by $\mathrm{SO}_{2} 9_{2,8}-9_{1,9}$ at $188654.973 \mathrm{MHz}$; and $\mathrm{HOCN} 12_{3,10}-11_{3,9}$ at the rest frequency $251528.269 \mathrm{MHz}$, which is likely affected by c- $\mathrm{C}_{3} \mathrm{H}_{2} 6_{2,5}-5_{1,4}$ at $251527.311 \mathrm{MHz}$ (Nummelin et al. 1998). Regarding HCNO, the number of predicted transitions is considerably minor in comparison to $\mathrm{HOCN}$, about 10 for the same interval of $E_{u}$. Similarly, the transition HCNO $12-11$ is identified at the rest frequency $275227.774 \mathrm{MHz}$, however it is likely affected by $\mathrm{SO}_{2} 15_{3,13}-15_{2,14}$ at $275240.184 \mathrm{MHz}$ (Loren \& Mundy 1984). Additionally, in the $\mathrm{SO}_{2}$ case, a factor that also makes difficult a positive identification is that the lines exhibit Lorentzian profiles, so that such emission could be more related to the outflow. In the $\mathrm{c}-\mathrm{C}_{3} \mathrm{H}_{2}$ case, the line exhibit a Gaussian profile as the HNCO lines, which could be more in agreement with the physical origin of the HNCO emission. In perspective, the 'puzzle' of the CHNO isomers in hot molecular cores is compelling, but theoretical and/or experimental approaches might be required for a better comprehension of such a chemical puzzle.

\subsection{Internal partition function of CHNO isomers}

The rotational-vibrational partition function of the isomers of CHNO (Section 4.2; McLean, Loew \& Berkowitz 1977) have been updated in this work according to Carvajal et al. (2019) and presented here altogether for their comparison.

Usually, the internal partition function is computed as a direct sum of a considerable number of rovibrational energies in order to reach a convergence at any temperature. Unfortunately, the spectroscopic data for the isomers of CHNO is still insufficient. Then, we have to compute the partition function adopting some suitable approximations that were already validated (see e.g. Carvajal et al. 2019, and references therein). Thus, the molecular partition functions for $\mathrm{CHNO}$ isomers are computed as a product of the rotational partition function $Q_{\text {rot }}(T)$ and of the vibrational contribution $Q_{\text {vib }}(T)$ :

$Q_{\mathrm{rv}}(T) \approx Q_{\mathrm{rot}}(T) Q_{\mathrm{vib}}(T)$, 
where the rotational contribution is computed as a direct sum whether the convergence is reached with the available spectroscopic data. In the particular cases of the CHNO isomers, the rotational spectroscopic information is available in the CDMS catalogue and the predicted rotational energies in the vibrational ground state can be computed with Pickett's code (Pickett 1991). Otherwise, the approximation of the classical partition function can be a suitable alternative to provide the rotational partition function for sources at not very low temperatures (Herzberg 1991) besides being a useful tool to test the convergence of the direct sum at higher temperatures (Carvajal et al. 2019). In the particular cases of CHNO isomers, the classical partition function values turn out to be adequate at temperatures higher than $20 \mathrm{~K}$. However, the addition of some simple correction terms to the classical partition function can improve this approximation considerably (Wells \& Raston 2020). For all the CHNO isomers, the nuclear spin statistical weights are the same and, in this work, they are considered as 1.

Concerning the vibrational partition functions for the CHNO isomers, these cannot be obtained as a direct sum because there are not enough experimental or calculated vibrational energies at disposal. Therefore, the harmonic approximation (Herzberg 1991) has been used to estimate the vibrational contribution of the partition function and therefore it is enough to know the vibrational fundamental frequencies. If any bending degree of freedom for these four isomers was susceptible to undergo a strong anharmonicity caused, e.g. by a large amplitude motion, the vibrational partition function could be split into two contributions (see e.g. Carvajal et al. 2019, and references therein): the one derived from small amplitude vibrations and another coming from the large amplitude vibrational modes. The vibrational partition function from the small amplitude vibrations can be computed with the harmonic approximation whereas the large amplitude contribution should be calculated as a direct sum using the predicted energies of the large amplitude bending states (Favre et al. 2014), which could be calculated with any approach conceived for the analysis of nonrigid molecules (see e.g. Jensen 1983; Khalouf-Rivera, Pérez-Bernal \& Carvajal 2020).

Table 2 provides, for the four CHNO isomers, the results of the vibrational contribution in the harmonic approximation $\left(Q_{\mathrm{vib}}^{\text {harm }}\right)$ of the internal partition function $\left(Q_{\mathrm{rv}}\right)$, the internal partition function computed in the present work by means of equation (4), the rotational partition function in the CDMS catalogue computed as a direct sum (Endres et al. 2016), and the relative differences between the values reported in the present work and in the CDMS for the temperature range from 2.725 to $500 \mathrm{~K}$. In addition, the uncertainties of the internal partition functions have been included for the four CHNO isomers. These have been estimated upwardly considering large uncertainties for the rotational and vibrational fundamental energies at $100 \mathrm{MHz}$ and $1 \mathrm{~cm}^{-1}$, respectively. In general, at temperatures above $20 \mathrm{~K}$, the relative difference between the partition function values obtained in the present work and those in CDMS catalogue are substantially larger than the upward estimate of the uncertainty of the internal partition function.

From Table 2, it can be observed that the rotational contributions to the internal partition functions of the four isomers have similar values at the lowest temperatures, e.g. down to $T=10 \mathrm{~K}$. For higher temperatures, above $T=225 \mathrm{~K}$, the difference between the rotational partition function of fulminic acid and the values of the other three isomers (isocyanic acid, cyanic acid, and isofulminic acid), with more similar values, becomes larger. This could be explained because of the structure of fulminic acid is linear and of the other have a near-prolate asymmetric top structure. Nevertheless, the internal partition functions of isocyanic acid, cyanic acid, and isofulminic acid become completely different at higher temperatures despite the fact that these three isomers have a bent structure. This stems from the differences among the vibrational partition functions of the bent isomers.

In Table 2, the weight of the vibrational contribution to the internal partition function can also be assessed for the set of the four CHNO isomers at different temperatures. The differences between the internal partition function and the rotational partition function become noteworthy (more than 1 per cent) at temperatures higher than $225 \mathrm{~K}$ for $\mathrm{HNCO}, 150 \mathrm{~K}$ for $\mathrm{HOCN}$, and $T=75 \mathrm{~K}$ for $\mathrm{HCNO}$ and HONC. Even when the vibrational partition function has been calculated with the harmonic approximation and, in some cases, using fundamentals, the values of this approximation, although underestimated, are in good agreement with the direct sum values (Carvajal et al. 2019).

As supplementary material, the rotational, vibrational, and rovibrational partition function calculated in the present work from 1 to $500 \mathrm{~K}$ (in intervals of $1 \mathrm{~K}$ ) is also provided for the following isomers: cyanic acid (HOCN); fulminic acid (HCNO); and isofulminic acid (HONC). For isocyanic acid (HNCO), same material can be found elsewhere (Carvajal et al. 2019).

In Appendix A, we will give some details about the calculation of the partition function for the four CHNO isomers.

\subsection{Chemical modelling with NAUTILUS}

In order to better understand the chemical pathways involved in the production and destruction of HNCO, we used the NAUTILUS code (Ruaud et al. 2016) to carry out a time-dependent chemical model. NAUTILUS allows to simulate and study grain- and gas-phase chemical processes for instance in hot and cold cores (Semenov et al. 2010; Reboussin et al. 2014; Ruaud et al. 2015, 2016). The predictions of the model include the time evolution of the chemical abundances for a given set of physical and chemical parameters. For the solid-state chemistry, it considers mantle and surface as chemically active, following the formalism of Hasegawa \& Herbst (1993) and the experimental results of Ghesquière et al. (2015). In this sense, the code can perform a three-phase (gas plus grain mantle and surface) time-dependant simulation of the chemistry in hot cores, including chemical reactions in both gas and solid phases (Ruaud et al. 2015).

This approach is interesting for our work since most of HNCO may be trapped in icy grains, as suggested by Altwegg et al. (2020). The grain chemistry of the code considers the standard direct photodissociation by photons along with the photodissociation induced by secondary UV photons, as described by Prasad \& Tarafdar (1983), which are effective processes on the surface and mantle of the grains. The network of chemical reactions used by the code is presented on the KInetic Database for Astrochemistry ${ }^{11}$ catalogue (Wakelam et al. 2015).

The first and simplest simulation to perform is a zero-dimensional, i.e. total density, gas temperature, and other physical condition are uniform within the considered core and throughout the simulation time. There is no structure evolution in this case. Once our source present a shell-like structure, a more elaborated structure model is necessary to be simulated together with NAUTILUS in order to

\footnotetext{
${ }^{11}$ http://kida.obs.u-bordeaux1.fr/
} 
Table 2. Vibrational and rotational-vibrational partition function for CHNO isomers: isocyanic acid (HNCO); cyanic acid (HOCN); fulminic acid (HCNO); and isofulminic acid (HONC). Comparison between the values obtained in the present study and those published in CDMS catalogue ${ }^{a}$.

\begin{tabular}{|c|c|c|c|c|c|c|c|c|}
\hline \multirow[b]{2}{*}{$T(\mathrm{~K})$} & \multicolumn{4}{|c|}{$\mathrm{HNCO}^{b}$} & \multicolumn{4}{|c|}{$\mathrm{HOCN}$} \\
\hline & $Q_{\mathrm{vib}}^{\text {harm } c}$ & $Q_{\mathrm{rv}}(\text { Carvajal et al. 2019 })^{d}$ & $Q(\mathrm{CDMS})^{e}$ & Rel. diff. $(\%)^{f}$ & $Q_{\text {vib }}^{\text {harm } c}$ & $Q_{\mathrm{rv}}(\text { Present work })^{d, g}$ & $Q(\mathrm{CDMS})^{e}$ & Rel. diff. $(\%)^{f}$ \\
\hline 2.725 & 1.000000 & $5.5129(50)$ & 5.51 & 0.00 & 1.000000 & $5.7601(52)$ & 5.7601 & 0.00 \\
\hline 9.375 & 1.000000 & $18.4493(36)$ & 18.45 & 0.00 & 1.000000 & $20.1244(37)$ & 20.1244 & 0.00 \\
\hline 18.750 & 1.000000 & $42.8295(30)$ & 42.83 & 0.00 & 1.000000 & $50.8161(32)$ & 50.8159 & 0.00 \\
\hline 37.500 & 1.000000 & $117.3053(27)$ & 117.30 & 0.01 & 1.000000 & $142.1565(12)$ & 142.1413 & 0.01 \\
\hline 225.000 & 1.048753 & $1827.01(36)$ & 1742.43 & 4.63 & 1.114711 & $2328.9(22)$ & 2087.4819 & 10.37 \\
\hline 300.000 & 1.144368 & $3080.9(12)$ & 2695.34 & 12.51 & 1.269376 & 4083.1(48) & 3217.0765 & 21.21 \\
\hline \multirow[t]{2}{*}{500.000} & 1.667813 & $9690.5(89)$ & 5866.52 & 39.46 & 1.984226 & 13733.(23) & - & - \\
\hline & \multicolumn{4}{|c|}{$\mathrm{HCNO}$} & \multicolumn{4}{|c|}{ HONC } \\
\hline$T(\mathrm{~K})$ & $Q_{\mathrm{vib}}^{\text {harm } c}$ & $Q_{\mathrm{rv}}(\text { Present work })^{d}$ & $Q(\mathrm{CDMS})^{e}$ & Rel. diff. $(\%)^{f}$ & $Q_{\mathrm{vib}}^{\text {harm } c}$ & $Q_{\mathrm{rv}}(\text { Present work })^{d, h}$ & $Q(\mathrm{CDMS})^{e}$ & Rel. diff. $(\%)^{f}$ \\
\hline 2.725 & 1.000000 & $5.2981(49)$ & 5.2980 & 0.00 & 1.000000 & $5.5525(50)$ & 5.5307 & 0.39 \\
\hline 75.000 & 1.027795 & $140.402(52)$ & 136.6050 & 2.70 & 1.007476 & $405.760(45)$ & 401.5499 & 1.04 \\
\hline 150.000 & 1.296184 & $353.73(63)$ & 272.9036 & 22.85 & 1.130785 & $1286.6(11)$ & 1135.8099 & 11.72 \\
\hline 225.000 & 1.842269 & $753.9(21)$ & 409.2300 & 45.72 & 1.393281 & 2911.3(48) & 2088.3400 & 28.27 \\
\hline 300.000 & 2.707133 & $1477.0(53)$ & 545.5841 & 63.06 & 1.764826 & $5676.3(125)$ & 3218.5016 & 43.30 \\
\hline 500.000 & 7.363415 & $6695(31)$ & - & - & 3.328126 & $23019 .(129)$ & - & - \\
\hline
\end{tabular}

${ }^{a}$ The nuclear spin degeneracy is considered as 1 .

${ }^{b}$ Result from Carvajal et al. (2019).

${ }^{c}$ The vibrational partition function is computed with the harmonic approximation. For more details, see the text.

${ }^{d} Q_{\mathrm{rv}}=Q_{\mathrm{rot}}$ (direct sum) $Q_{\mathrm{vib}}^{\mathrm{harm}}$ unless noted otherwise. An upward estimate of the uncertainties is given in parentheses in units of the last quoted digits. For more details, see the text.

${ }^{e}$ Rotational partition function computed as a direct sum with no vibrational contribution. Their values are reported in CDMS catalogue (Endres et al. 2016).

${ }^{f}$ Relative difference of the partition function given in the present study with respect to the one reported in CDMS catalogue.

${ }^{g}$ The rotational contribution of the partition function is computed with a direct sum up to $\mathrm{T}=18.75 \mathrm{~K}$ inclusive. From $37.50 \mathrm{~K}$ forward, the rotational partition function is obtained with the classical approximation. For more details, see the text.

${ }^{h}$ The rotational contribution of the partition function is computed with a direct sum except for $\mathrm{T}=500 \mathrm{~K}$, which is obtained with the classical approximation. For more details, see the text.

Table 3. Initial abundances assumed for the NAUTILUS model (extracted from Vidal \& Wakelam (2018)). References are: 1. Wakelam \& Herbst (2008); 2. Jenkins (2009); 3. Hincelin et al. (2011); 4. low-metal abundances from Graedel, Langer \& Frerking (1982); 5. depleted value from Neufeld, Wolfire \& Schilke (2005).

\begin{tabular}{llcccc}
\hline Element & $\mathrm{n}_{i} / \mathrm{n}_{H}{ }^{a}$ & Ref. & Element & $\mathrm{n}_{i} / \mathrm{n}_{H}{ }^{a}$ & Ref. \\
\hline $\mathrm{H}_{2}$ & 0.5 & & $\mathrm{He}$ & $9.0(-2)$ & 1 \\
$\mathrm{~N}$ & $6.2(-5)$ & 2 & $\mathrm{O}$ & $2.4(-4)$ & 3 \\
$\mathrm{C}^{+}$ & $1.7(-4)$ & 2 & $\mathrm{~S}^{+}$ & $1.5(-5)$ & 2 \\
$\mathrm{Fe}^{+}$ & $3.0(-9)$ & 4 & $\mathrm{Si}^{+}$ & $8.0(-9)$ & 4 \\
$\mathrm{Na}^{+}$ & $2.0(-9)$ & 4 & $\mathrm{Mg}^{+}$ & $7.0(-9)$ & 4 \\
$\mathrm{Cl}^{+}$ & $1.0(-9)$ & 4 & $\mathrm{P}^{+}$ & $2.0(-10)$ & 4 \\
$\mathrm{~F}$ & $6.7(-9)$ & 5 & & & \\
\hline
\end{tabular}

${ }^{a}$ Abundances given in the format a(b) representing a $\times 10^{b}$.

represent the HNCO abundances more accurately. Nevertheless, this approximation can furnish the first insights in the chemistry of G331. The initial elemental abundances for the core were selected from the recent values used by Vidal \& Wakelam (2018) and are displayed in Table 3. They represent an intermediate-metal abundance case, in which all the abundances are the same as the low-metal one except that the amount of the element sulphur is raised to $1.5 \times 10^{-5}$ compared with $\mathrm{H}_{2}$ (Wakelam, Herbst \& Selsis 2006). Concerning parameters such as the visual extinction, we assumed a typical value of 10 mag for a dark cloud. The gas and dust temperatures were considered the same in the simulations.

Several simulations were tested for a range of density and temperature of $10^{6}-10^{8} \mathrm{~cm}^{-3}$ and $50-200 \mathrm{~K}$, respectively (see Canelo 2020, for more details). The best-fitting model is obtained adopting a density of $1 \times 10^{7} \mathrm{~cm}^{-3}$ and a temperature of $60 \mathrm{~K}$, and is presented in Fig. 8. The standard cosmic ray ionization rate for $\mathrm{H}_{2}$ $\left(\zeta_{\mathrm{H}_{2}}\right)$ is $1.3 \times 10^{-17} \mathrm{~s}^{-1}$ (Wakelam et al. 2015; Ruaud et al. 2016), which is a key parameter for the gas-grain models since cosmic rays induce desorption processes in which chemical species pass to the gas phase from grain surfaces (Hasegawa \& Herbst 1993; Ruaud et al. 2015). Taking into account that the cosmic ray ionization might vary in interstellar gas clouds, e.g. $0.6-6 \times 10^{-17} \mathrm{~s}^{-1}$ (Van der Tak \& van Dishoeck 2000; Dalgarno 2006), we also tested models considering $\zeta_{\mathrm{H}_{2}}=1.3 \times 10^{-16}$ and $1.3 \times 10^{-18} \mathrm{~s}^{-1}$, the results are shown in Fig. 8. The main difference between the models is the plateau of the abundance intensity peaks and, consequently, the position of the maximum value. The maximum abundances obtained were $1.86,4.19$, and $5.48 \times 10^{-8}$, respectively. Although these abundances are a bit higher than those obtained from the rotational diagrams, the model at $60 \mathrm{~K}$ is the only one that can reproduce the abundance range of G331 and also the excitation temperature derived for HNCO, although the final adopted density is slightly higher than the core density derived by Hervías-Caimapo et al. (2019; see Section 1). 


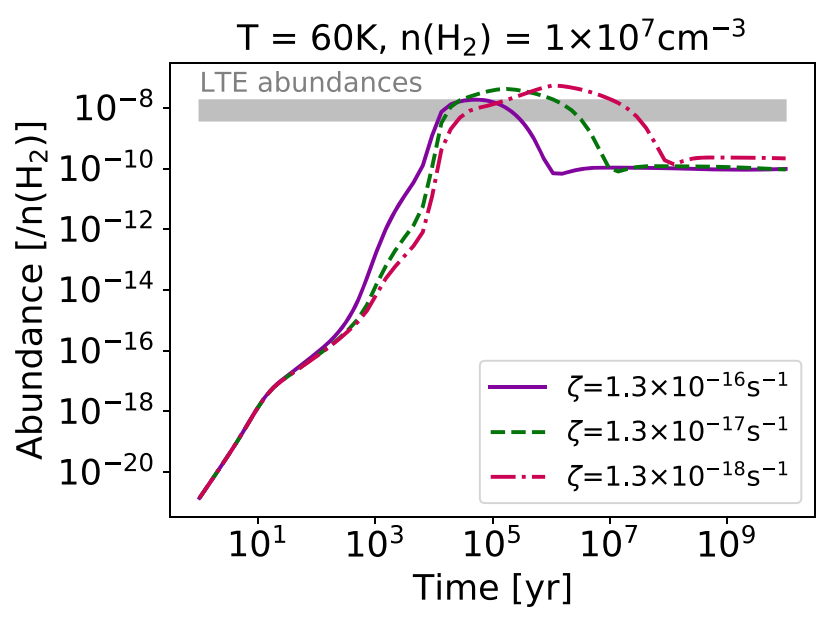

Figure 8. Best-fitting time evolution of the HNCO abundances simulated with NAUTILUS. We assumed a gas and grain temperatures of $60 \mathrm{~K}$, and density of $10^{7} \mathrm{~cm}^{-3}$. The cosmic ray ionization rate $(\zeta)$ was varied in $1.3 \times 10^{-16}$, $1.3 \times 10^{-17}$, and $1.3 \times 10^{-18} \mathrm{~s}^{-1}$. The grey strip indicates the range of the derived LTE abundances.

From Fig. 8, it can be seen that the balance of the chemical reaction and chemical equilibrium are highly dependent on the cosmic ionization rate. Higher rates induce more chemical reactions (as expected, as it is a more significant energy source) that last a shorter period of time. Maximum abundances are also lower but the chemical equilibrium occurs faster. Considering $\zeta=1.3 \times 10^{-17} \mathrm{~s}^{-1}$, the chemical age could be between $3 \times 10^{4-6}$ yr with a maximum simulated abundance around $10^{5} \mathrm{yr}$. The other limiting values of $\zeta$ presented the same abundance $1.4 \times 10^{-8}$ derived for G331 at $10^{5} \mathrm{yr}$, approximately. These models could suggest that $10^{5} \mathrm{yr}$ may be a plausible chemical age for G331. Hot cores and hot corinos, in general, are expected to have a dynamical age up to $10^{4-5} \mathrm{yr}$, as obtained for IRAS 16293-2422 (Hernández-Gómez et al. 2019) and G10.47+0.03 (Gorai et al. 2020), for example. Within this scenario, the chemical age derived for G331 is also in agreement with most of the objects of Fig. 7. The molecular flow of the evolved star $\mathrm{OH} 231.8+4.2$ has an estimated dynamical age of $800 \mathrm{yr}$, but this source is in a later evolutionary stage. Nevertheless, we cannot conclude that HNCO could be used as a chemical clock. Our simulations are zero-dimensional and this molecule can be highly dependent on the initial parameters (Hernández-Gómez et al. 2019). Moreover, the chemical age obtained with NAUTILUS is, at least, one order of magnitude larger than the estimated dynamical age of G331 ( $\sim 2000$ yr, Merello et al. 2013b).

In these simulations, the code uses two formation pathways which lead to HNCO by means of gas-grain processes. The whole mechanism suggest that $\mathrm{HNCO}$ is initially formed on the surface of grains, via $\mathrm{H}+\mathrm{OCN} \rightarrow \mathrm{HNCO}$, then it might pass from the solid (s) to the gaseous ( $\mathrm{g}$ ) phase through sublimation processes, via $\mathrm{HNCO}(\mathrm{s}) \rightarrow \mathrm{HNCO}(\mathrm{g})$. They are enough to reproduce the observed abundances in this temperature regime and with a zerodimension simulation, but the code returns a greater time-scale to do it so. Furthermore, higher temperatures presented a drastic decrease in the simulated abundance. For a more robust model, with structure evolution and time evolution of physical conditions, other reactions may have to be included in the code, if their rate coefficients are available. One example is the reaction $\mathrm{NH}+\mathrm{CO} \rightarrow \mathrm{HNCO}$, normally proposed as the main pathway to form $\mathrm{HNCO}$ in analogous mixtures of interstellar ice processed by proton or UV radiation (Fedoseev et al. 2015). It is also possible that the incorporation of this reaction in the code may lead to a chemical age similar to the previously derived dynamical age of G331.

\section{CONCLUSIONS}

In this paper, we reported the observation of 42 emission lines of the potential prebiotic molecule $\mathrm{HNCO}$ in the hot molecular core G331. This core was observed with the APEX telescope covering the interval frequency $160-355 \mathrm{GHz}$. The HNCO transitions are distributed into their $K_{a}$-ladder numbers of 0,1 , and 2 . The $K_{a}=0$ transitions presented the highest fluxes while the $K_{a}=2$ transitions showed the lowest ones, appearing as spectral pairs partially resolved. Such trends are expected from the different $K_{a}$-ladder numbers, in which the spectral pairs of each ladder get a better separation as the frequency increase. Furthermore, the observations allowed to see a particular spectral profile with extended tails in some HNCO lines, as in the HNCO $J=8-7$ and $J=15-14$ transitions. The HNCO emission can be responsible for about 80 per cent of the entire flux, while the wing could be an effect of the outflow or a contaminant emission.

With LTE rotational diagrams, we obtained the HNCO excitation temperature and column density of $T_{e x}=59.4 \pm 2.3 \mathrm{~K}$ and $N(\mathrm{HNCO})$ $=(3.1 \pm 0.4) \times 10^{15} \mathrm{~cm}^{-2}$, without the opacity correction, and $T_{e x}=$ $58.8 \pm 2.7 \mathrm{~K}$ and $N(\mathrm{HNCO})=(3.7 \pm 0.5) \times 10^{15} \mathrm{~cm}^{-2}$, considering beam dilution effects. This value of temperature could indicate that HNCO molecules are located in the external and colder regions of G331, according to the shell-like structure model of G331. We also derived the HNCO relative abundances by considering two main values, so that it was obtained values in the interval of $(3.8 \pm 0.5) \times$ $10^{-9}<[\mathrm{HNCO}]<(1.4 \pm 0.2) \times 10^{-8}$. We compared our column densities and abundances of HNCO with values from other hot molecular cores and evolved objects, and our results are in agreement with most of the objects.

In addition, the internal partition functions of the four $\mathrm{CHNO}$ isomers (isocyanic acid, HNCO; cyanic acid, $\mathrm{HOCN}$; fulminic acid, HCNO; and isofulminic acid, HONC) have been updated with the vibrational contribution and their values are provided from 1 to $500 \mathrm{~K}$ in intervals of $1 \mathrm{~K}$ in the supplementary material. We think that this update is relevant for the estimate of abundances of the four CHNO isomers in surveys at temperatures higher than $225 \mathrm{~K}$ for $\mathrm{HNCO}$, $150 \mathrm{~K}$ for $\mathrm{HOCN}$, and $T=75 \mathrm{~K}$ for $\mathrm{HCNO}$ and HONC.

Finally, we simulated the chemistry of HNCO with the threephase time-dependent code NAUTILUS. We used a zero-dimensional simulation with a temperature of $60 \mathrm{~K}$ that represented our derived abundances without the need to add more grain-phase reactions to the NAUTILUS database. We also varied the cosmic ionization rate to better comprehend the influence of this physical parameter in the simulations. From the models, we could also suggest a chemical age around $10^{5} \mathrm{yr}$ for G331, which is higher than estimated dynamical age. One explanation for this difference could be the absence of a key reaction to form $\mathrm{HNCO}$ in the code.

\section{ACKNOWLEDGEMENTS}

The authors thank the anonymous referee for the useful comments that improved the article. CMC acknowledges the support of CNPq, Conselho Nacional de Desenvolvimento Científico e Tecnológico Brazil, process number 141714/2016-6. This study was financed in part by the Coordenação de Aperfeiçoamento de Pessoal de Nível Superior - Brasil (CAPES) - Finance Code 001. LB acknowledges support from CONICYT (Comisión Nacional de Investigación Científica 
y Tecnológica) project Basal AFB-170002. EM acknowledges support from the Brazilian agencies FAPESP (Fundação de Amparo à Pesquisa do Estado de São Paulo, grant 2014/22095-6) and CNPq (grant 150465/2019-0). MC acknowledges the financial support from the European Union's Horizon 2020 research and innovation program under the Marie Skłodowska-Curie grant agreement no 872081; from the Spanish National Research, Development, and Innovation plan (RDI plan) under the project PID2019-104002GB-C21; the Consejería de Conocimiento, Investigación y Universidad, Junta de Andalucía and European Regional Development Fund (ERDF), ref. SOMM17/6105/UGR; the Ministerio de Ciencia, Innovación y Universidades (ref. COOPB20364); and by the Centro de Estudios Avanzados en Física, Matemáticas y Computación (CEAFMC) of the University of Huelva.

\section{DATA AVAILABILITY}

The data underlying this article will be shared on reasonable request to the corresponding author.

\section{REFERENCES}

Albert S., Winnewisser M., Winnewisser B., 1996, Ber. Bunsenges. Phys. Chem., 100, 1876

Altwegg K. et al., 2020, Nat. Astron., 4, 533

Armstrong T., Loren R. B., 1984, Tech. Rep. AST, 8116403-1

Bally J., 2016, ARA\&A, 54, 491

Belitsky V. et al., 2018, A\&A, 612, A23

Bisschop S. E., Jørgensen J. K., van Dishoeck E. F., de Wachter E. B. M., 2007, A\&A, 465, 913

Biver N. et al., 2006, A\&A, 449, 1255

Bronfman L., Garay G., Merello M., Mardones D., May J., Brooks K. J., Nyman L.-Å., Güsten R., 2008, ApJ, 672, 391

Brunken S., Gottlieb C., McCarthy M., Thaddeus P., 2009, ApJ, 697, 880

Brünken S., Belloche A., Martín S., Verheyen L., Menten K. M., 2010, A\&A, 516, A109

Canelo C. M., 2020, PhD thesis, IAG-USP, Brasil doi:https://doi.org/10.116 06/T.14.2020.tde-03022021-131439

Carvajal M., Favre C., Kleiner I., Ceccarelli C., Bergin E. A., Fedele D., 2019, A\&A, 627, A65

Churchwell E., Wood D., Myers P. C., Myers R. V., 1986, ApJ, 305, 405

Crovisier J., 1998, Faraday Discuss., 109, 437

Dalgarno A., 2006, Proc. Natl. Acad. Sci., 103, 12269

Dobrijevic M., Hébrard E., Loison J. C., Hickson K. M., 2014, Icarus, 228, 324

Dumke M., Mac-Auliffe F., 2010, in Proc. SPIE, Vol. 7737. p. 77371J

Duronea N. U. et al., 2019, MNRAS, 489, 1519

East A. L. L., Johnson C. S., Allen W. D., 1993, J. Chem. Phys., 98, 1299

Endres C. P., Schlemmer S., Schilke P., Stutzki J., Müller H. S. P., 2016, J. Mol. Spectrosc., 327, 95

Favre C. et al., 2014, ApJS, 215, 25

Fedoseev G., Ioppolo S., Zhao D., Lamberts T., Linnartz H., 2015, MNRAS, 446, 439

Ghesquière P., Mineva T., Talbi D., Theulé P., Noble J. A., Chiavassa T., 2015, Phys. Chem. Chem. Phys. (Inc. Faraday Trans.), 17, 11455

Goldsmith P. F., Langer W. D., 1999, ApJ, 517, 209

Gorai P., Bhat B., Sil M., Mondal S. K., Ghosh R., Chakrabarti S. K., Das A., 2020, ApJ, 895, 86

Graedel T. E., Langer W. D., Frerking M. A., 1982, ApJS, 48, 321

Greaves J. S., White G. J., 1991, A\&AS, 91, 237

Güsten R., Nyman L. Å., Schilke P., Menten K., Cesarsky C., Booth R., 2006, A\&A, 454, L13

Hasegawa T. I., Herbst E., 1993, MNRAS, 263, 589

He Y.-X. et al., 2021, ApJS, 253, 2

Herbst E., van Dishoeck E. F., 2009, ARA\&A, 47, 427
Hernández-Gómez A., Sahnoun E., Caux E., Wiesenfeld L., Loinard L., Bottinelli S., Hammami K., Menten K. M., 2019, MNRAS, 483, 2014

Hervías-Caimapo C. et al., 2019, ApJ, 872, 200

Herzberg G., 1991, Spectra and Molecular Structure: II. Infrared and Raman Spectra of Polyatomic Molecules. Krieger Pub. Co., Malabar, Florida

Hincelin U., Wakelam V., Hersant F., Guilloteau S., Loison J. C., Honvault P., Troe J., 2011, A\&A, 530, A61

Hocking W. H., Gerry M. C. L., Winnewisser G., 1972, ApJ, 174, L93

Hocking W. H., Gerry M. C. L., Winnewisser G., 1974, ApJ, 187, L89

Hocking W. H., Gerry M. C. L., Winnewisser G., 1975, Can. J. Phys., 53, 1869

Jenkins E. B., 2009, ApJ, 700, 1299

Jensen P., 1983, J. Mol. Spectrosc., 101, 422

Jewell P. R., Hollis J. M., Lovas F. J., Snyder L. E., 1989, ApJS, 70, 833

Jones L. H., Badger R. M., 1950, J. Chem. Phys., 18, 1511

Jones L. H., Shoolery J. N., Shulman R. G., Yost D. M., 1950, J. Chem. Phys., 18,990

Khalouf-Rivera J., Pérez-Bernal F., Carvajal M., 2020, J. Quant. Spectrosc. Radiat. Transfer, 261, 107436

Lapinov A. V., Golubiatnikov G. Y., Markov V. N., Guarnieri A., 2007, Astron. Lett., 33, 121

Li J., Wang J. Z., Gu Q. S., Zheng X. W., 2013, A\&A, 555, A18

Ligterink N. F. W., Terwisscha van Scheltinga J., Taquet V., Jørgensen J. K., Cazaux S., van Dishoeck E. F., Linnartz H., 2018, MNRAS, 480, 3628

Lis D. C. et al., 1997, Icarus, 130, 355

López-Sepulcre A. et al., 2015, MNRAS, 449, 2438

López-Sepulcre A., Balucani N., Ceccarelli C., Codella C., Dulieu F., Theulé P., 2019, ACS Earth Space Chem., 3, 2122

Loren R. B., Mundy L. G., 1984, ApJ, 286, 232

Lovas F. J., 2004, J. Phys. Chem. Ref. Data, 33, 177

MacDonald G. H., Gibb A. G., Habing R. J., Millar T. J., 1996, A\&AS, 119, 333

Marcelino N., Cernicharo J., Tercero B., Roueff E., 2009, ApJ, 690, L27

Martín S., Mauersberger R., Martín-Pintado J., Henkel C., García-Burillo S., 2006, ApJS, 164, 450

Martín S., Requena-Torres M. A., Martín-Pintado J., Mauersberger R., 2008, ApJ, 678, 245

Martín S., Martín-Pintado J., Mauersberger R., 2009, ApJ, 694, 610

Martín-Doménech R., Rivilla V. M., Jiménez-Serra I., Quénard D., Testi L., Martín-Pintado J., 2017, MNRAS, 469, 2230

McLean A., Loew G., Berkowitz D., 1977, J. Mol. Spectrosc., 64, 184

Meier D. S., Turner J. L., 2005, ApJ, 618, 259

Mendoza E., Lefloch B., López-Sepulcre A., Ceccarelli C., Codella C., Boechat-Roberty H. M., Bachiller R., 2014, MNRAS, 445, 151

Mendoza E. et al., 2018, ApJ, 853, 152

Merello M., Bronfman L., Garay G., Nyman L.-Å., Evans, Neal J. I., Walmsley C. M., 2013a, ApJ, 774, 38

Merello M., Bronfman L., Garay G., Lo N., Evans N. J. II, Nyman L. Å., Cortés J. R., Cunningham M. R., 2013b, ApJ, 774, L7

Mladenovic M., Lewerenz M., 2008, Chem. Phys., 343, 129

Mladenovic M., Lewerenz M., McCarthy M., Thaddeus P., 2009, J. Chem. Phys., 131, 174308

Neufeld D. A., Wolfire M. G., Schilke P., 2005, ApJ, 628, 260

Nguyen-Q-Rieu, Henkel C., Jackson J. M., Mauersberger R., 1991, A\&A, 241, L33

Niedenhoff M., Yamada K. M. T., Belov S. P., Winnewisser G., 1995, J. Mol. Spectrosc., 174, 151

Nummelin A., Bergman P., Hjalmarson Å., Friberg P., Irvine W. M., Millar T. J., Ohishi M., Saito S., 1998, ApJS, 117, 427

Pickett H. M., 1991, J. Mol. Spectrosc., 148, 371

Pickett H. M., Poynter R. L., Cohen E. A., Delitsky M. L., Pearson J. C., Müller H. S. P., 1998, J. Quant. Spectrosc. Radiat. Transfer, 60, 883

Prasad S. S., Tarafdar S. P., 1983, ApJ, 267, 603

Reboussin L., Wakelam V., Guilloteau S., Hersant F., 2014, MNRAS, 440, 3557

Ruaud M., Loison J. C., Hickson K. M., Gratier P., Hersant F., Wakelam V., 2015, MNRAS, 447, 4004

Ruaud M., Wakelam V., Hersant F., 2016, MNRAS, 459, 3756 
Schulze G., Koja O., Winnewisser B., Winnewisser M., 2000, J. Mol. Struct., 517/518, 307

Semenov D. et al., 2010, A\&A, 522, A42

Shimanouchi T., 2018, Molecular Vibrational Frequencies in NIST Chemistry WebBook. NIST Standard Reference Database Number 69. P.J. Linstrom and W.G. Mallard, National Institute of Standards and Technology, Gaithersburg MD, 20899, USA

Snyder L. E., Buhl D., 1972, ApJ, 177, 619

Snyder L. E. et al., 2005, ApJ, 619, 914

Su H., Kong F., Chen B., Huang M., Liu Y., 2000, J. Chem. Phys., 113, 1885

Sutton E. C., Blake G. A., Masson C. R., Phillips T. G., 1985, ApJS, 58, 341

Sutton E. C., Jaminet P. A., Danchi W. C., Blake G. A., 1991, ApJS, 77, 255

Takashi R., Tanaka K., Tanaka T., 1989, J. Mol. Spectrosc., 138, 450

Turner B. E., 1991, ApJS, 76, 617

Van der Tak F. F. S., van Dishoeck E. F., 2000, A\&A, 358, L79

Vassilev V. et al., 2008, A\&A, 490, 1157

Velilla Prieto L. et al., 2015, A\&A, 575, A84

Vidal T. H. G., Wakelam V., 2018, MNRAS, 474, 5575

Wakelam V., Herbst E., 2008, ApJ, 680, 371

Wakelam V., Herbst E., Selsis F., 2006, A\&A, 451, 551

Wakelam V. et al., 2015, ApJS, 217, 20

Wells T., Raston P., 2020, J. Mol. Spectrosc., 370, 111292

Winnewisser M., Winnewisser B., 1971, Z. Naturforsch., 26, 128

Wyrowski F., Schilke P., Walmsley C. M., 1999, A\&A, 341, 882

Zinchenko I., Henkel C., Mao R. Q., 2000, A\&A, 361, 1079

\section{APPENDIX A: CALCULATION OF THE PARTITION FUNCTIONS OF CHNO ISOMERS}

Here we are giving some details for the calculation of the rovibrational partition function for the isomers of $\mathrm{CHNO}$ isomers (isocyanic acid, cyanic acid, fulminic acid, and isofulminic acid).

\section{A1 Isocyanic acid (HNCO)}

Isocyanic acid is a near-prolate asymmetric top of which rotationalvibrational partition function has been previously calculated (Carvajal et al. 2019). The main results of this isomer have been included here to compare them with the internal partition functions of the other three isomers. Thus, here we only outline the details for its calculation.

The internal partition function has been computed from equation (4). The rotational partition function has been computed as a direct sum taking into consideration the rotational energies predicted up to $J=135$ and $\mathrm{K}_{a}=30$ from the Hamiltonian provided by Lapinov et al. (2007). The harmonic vibrational partition function has been calculated with the experimental vibrational fundamental frequencies recorded by East, Johnson \& Allen (1993).

\section{A2 Cyanic acid (HOCN)}

Cyanic acid is a bent molecule with a near-prolate asymmetric top structure of which rotational spectrum has been measured in the centimetre- and millimetre-wave windows (Brunken et al. 2009). This molecular species has no available experimental vibrational energies in the gas phase except the $v_{2}$ band ( $\mathrm{Su}$ et al. 2000; Shimanouchi 2018) but the complete set of fundamental frequencies have been obtained theoretically (Mladenovic \& Lewerenz 2008).

The values of the rotational partition function calculated as a direct sum and as a classical approximation are given in Table A1. The direct sum is obtained using the predicted rotational energy levels up to $J=72$ and $K_{a}=8$ (Brunken et al. 2009) provided in the
Table A1. Rotational partition function for cyanic acid (HOCN). Comparison between the direct sum values and the classical approximation $^{a}$.

\begin{tabular}{lrcc}
\hline$T(\mathrm{~K})$ & $Q_{\text {rot }}^{\text {approx }_{b}}$ & $\begin{array}{c}\text { HOCN } \\
Q_{\text {rot }} \\
\text { (direct sum) }^{c}\end{array}$ & $\begin{array}{c}\text { Rel. Diff. } \\
\text { (per cent) }^{d}\end{array}$ \\
\hline 2.725 & 2.78 & 5.76 & 51.66 \\
5.000 & 6.92 & 10.30 & 32.83 \\
9.375 & 17.77 & 20.12 & 11.70 \\
18.750 & 50.26 & 50.82 & 1.09 \\
37.500 & 142.16 & 142.14 & -0.01 \\
75.000 & 402.08 & 401.38 & -0.18 \\
150.000 & 1137.25 & 1135.36 & -0.17 \\
225.000 & 2089.27 & 2087.44 & -0.09 \\
300.000 & 3216.64 & 3215.89 & -0.02 \\
500.000 & 6921.10 & 6863.28 & -0.84 \\
\hline
\end{tabular}

${ }^{a}$ Nuclear spin degeneracy is considered as 1 .

${ }^{b}$ Rotational partition function computed with the classical approximation using the rotational constants from Brunken et al. (2009). ${ }^{c}$ Rotational partition function computed as a direct sum considering the predicted rotational energy levels up to $J=72$ and $\mathrm{K}_{a}=8$ (Brunken et al. 2009). The predicted rotational energies are also given in CDMS (Endres et al. 2016).

${ }^{d}$ Relative differences.

CDMS (Endres et al. 2016). The classical approximation is computed with the rotational constants from Brunken et al. (2009). In Table A1, it can be observed that the relative differences between the two procedures are relatively small for temperatures above $\mathrm{T}=18.75 \mathrm{~K}$. Nevertheless, it should be highlighted that, for temperatures from 37.5 to $300 \mathrm{~K}$, the direct sum results in slightly smaller values than the ones provided by the classical approximation. This fact could suggest that the direct sum has not been converged for this temperature interval. On the contrary, the direct sum has reached the convergence because, if this had not been reached at $\mathrm{T}=37.5 \mathrm{~K}$, the difference between the direct sum and the classical approximation would have been increased for higher temperatures. In addition, we have compared the values obtained through the direct sum in this work and the rotational partition function values provided in the CDMS (Endres et al. 2016), which are also reported in Table 2. It can be observed that the values of the direct sum in this work and those from CDMS, which only provides values up to $\mathrm{T}=300 \mathrm{~K}$, are practically the same. The relative difference between them is smaller than 0.00 per cent except for $\mathrm{T}=300 \mathrm{~K}$, which is of -0.04 per cent. Thus, it can be considered that the direct sum values of the rotational partition function have reached the convergence for all the temperature range up to $\mathrm{T}=300 \mathrm{~K}$. However, at $500 \mathrm{~K}$, according to the increasing difference between the direct sum and the classical approximation, we can state that the direct sum has not converged for this temperature. Therefore, even though the convergence of the direct sum seems to be reached up to $\mathrm{T}=300 \mathrm{~K}$, we will consider for the rotational partition function in equation (4) the values of the direct sum up to $\mathrm{T}=36 \mathrm{~K}$ inclusive and of the classical approximation from $\mathrm{T}=37 \mathrm{~K}$ up, according to the highest value.

The vibrational partition function has been obtained using the harmonic approximation from the $a b$ initio fundamental vibrational frequencies calculated with the $\operatorname{CCSD}(\mathrm{T}) / \mathrm{cc}-\mathrm{pVQZ}$ (all) level of theory (Mladenovic \& Lewerenz 2008). Their values are given in Table 2 together with the values of rovibrational partition function computed with equation (4) as well as those up to $300 \mathrm{~K}$ of the CDMS rotational partition function. The relative difference between 
Table A2. Rotational partition function for fulminic acid (HCNO). Comparison between the direct sum values and the classical approximation $^{a}$.

\begin{tabular}{lrrc}
\hline$T(\mathrm{~K})$ & $Q_{\text {rot }}^{\text {approx }} b$ & $\begin{array}{c}\text { HCNO } \\
Q_{\text {rot }} \\
\text { (directsum) }^{c}\end{array}$ & $\begin{array}{c}\text { Rel. diff. } \\
\text { (per cent) }^{d}\end{array}$ \\
\hline 2.725 & 4.950696 & 5.298078 & 6.56 \\
5.000 & 9.083845 & 9.424730 & 3.62 \\
9.375 & 17.032210 & 17.369697 & 1.94 \\
18.750 & 34.064420 & 34.400567 & 0.98 \\
37.500 & 68.128841 & 68.466594 & 0.49 \\
75.000 & 136.257682 & 136.605301 & 0.25 \\
150.000 & 272.515363 & 272.904169 & 0.14 \\
225.000 & 408.773045 & 409.230843 & 0.11 \\
300.000 & 545.030726 & 545.585087 & 0.10 \\
500.000 & 908.384544 & 909.229250 & 0.09 \\
\hline
\end{tabular}

${ }^{a}$ Nuclear spin degeneracy is considered as 1 .

${ }^{b}$ Rotational partition function computed with the classical approximation for linear molecules using the rotational constant from Winnewisser \& Winnewisser (1971).

${ }^{c}$ Rotational partition function computed as a direct sum considering the predicted rotational energy levels up to $J=90$ (Winnewisser \& Winnewisser 1971). The predicted rotational energies are also reported in the CDMS (Endres et al. 2016)

${ }^{d}$ Relative differences.

the results of this work and of CDMS become larger than 10 per cent at $225 \mathrm{~K}$ of temperature. Despite it is expected that the new values of the internal partition function are more precise, a more comprehensive list of experimental and predicted rovibrational data would be necessary to improve the partition function values as well as to get the convergence for the direct sum along all the interval up to $\mathrm{T}=500 \mathrm{~K}$.

In the supplementary material, it is provided the rotational (as a direct sum up to $\mathrm{T}=36 \mathrm{~K}$ and from this temperature forward as a classical approximation), vibrational and rovibrational partition function computed in the present work for cyanic acid (HOCN) up to $\mathrm{T}=500 \mathrm{~K}$ using a $1 \mathrm{~K}$ interval.

\section{A3 Fulminic acid (HCNO)}

Fulminic acid is a linear molecule of which experimental spectrum has been recorded in the millimetre-wave rotational and infrared ranges (Winnewisser \& Winnewisser 1971; Albert, Winnewisser \& Winnewisser 1996; Schulze et al. 2000). The internal partition function has been computed using equation (4) where the rotational partition function has been calculated as a direct sum and compared in Table A2 to the classical approximation for a linear molecule (Herzberg 1991).

The direct sum of the rotational partition function has been obtained considering the predicted rotational energies in the vibrational ground state up to $J=90$. The predictions have been calculated from the rotational Hamiltonian provided by Winnewisser \& Winnewisser (1971) although, in this particular case, they have also been reported in the CDMS (Endres et al. 2016). The classical approximation has been calculated from the rotational constant $B$ provided by Winnewisser \& Winnewisser (1971). In Table A2, it can be observed that the classical approximation has a good agreement with the direct sum from $\mathrm{T}=18.75 \mathrm{~K}$ up and, although slightly smaller, the difference decreases for higher temperatures.

The harmonic approximation of the vibrational partition function has been obtained using the experimental fundamental vibrational
Table A3. Rotational partition function for isofulminic acid (HONC). Comparison between the direct sum values and the classical approximation $^{a}$.

\begin{tabular}{|c|c|c|c|}
\hline$T(\mathrm{~K})$ & $Q_{\mathrm{rot}}^{\text {approx } b}$ & $\begin{array}{c}\text { HONC } \\
Q_{\text {rot }} \\
(\text { directsum })^{c}\end{array}$ & $\begin{array}{l}\text { Rel. diff. } \\
\text { (per cent })^{d}\end{array}$ \\
\hline 2.725 & 2.782829 & 5.552537 & 49.88 \\
\hline 5.000 & 6.916585 & 9.941600 & 30.43 \\
\hline 9.375 & 17.757982 & 19.740254 & 10.04 \\
\hline 18.750 & 50.227159 & 50.905466 & 1.33 \\
\hline 37.500 & 142.063859 & 142.724545 & 0.46 \\
\hline 75.000 & 401.817274 & 402.748482 & 0.23 \\
\hline 150.000 & 1136.510876 & 1137.826802 & 0.12 \\
\hline 225.000 & 2087.903800 & 2089.514569 & 0.08 \\
\hline 300.000 & 3214.538189 & 3216.363948 & 0.06 \\
\hline 500.000 & 6916.584928 & 6911.736014 & -0.07 \\
\hline
\end{tabular}

energies (Albert et al. 1996; Schulze et al. 2000; Shimanouchi 2018). These values are given in Table 2 together with the values of the internal partition function, written as product of the direct sum of the rotational contribution and harmonic approximation of the vibrational contribution, and the CDMS rotational partition function calculated as a direct sum (Endres et al. 2016). In addition, the relative difference between the internal partition function obtained in this work and the CDMS partition function is also given. It can be noted that the relative difference is larger than 20 per cent at temperatures higher than $150 \mathrm{~K}$. CDMS does not provide the partition function at $\mathrm{T}=500 \mathrm{~K}$

In the supplementary material, it is reported the rotational (as a direct sum), vibrational, and rovibrational partition function computed in the present work for fulminic acid (HCNO) up to $\mathrm{T}=500 \mathrm{~K}$ using a $1 \mathrm{~K}$ interval.

\section{A4 Isofulminic acid (HONC)}

Isofulminic acid is the CHNO isomer with less spectroscopic information. This molecule is a non-linear asymmetric top molecule close to the prolate limit. Up to the present, the only experimental spectroscopic paper about this species provides some microwave data as well as ab initio calculations of the rotational parameters and the fundamental vibrational energies (Mladenovic et al. 2009).

Despite the lack of data, the rotational partition function has been calculated as a direct sum and this has been compared to the values given by the classical approximation (see Table A3). The direct sum has been calculated from the rotational energies up to $J=80$ and $K_{a}=20$ predicted with SPCAT code (Pickett 1991) using the experimental value of the rotational constant $B$ and the $a b$ initio values of the rotational constants $A$ and $C$ (Mladenovic et al. 2009). These latter parameters have also been used for the classical approximation. The comparison between the direct sum and the classical approximation for the rotational partition function is also provided in Table A3. From temperatures above $18.75 \mathrm{~K}$, the relative difference is rather small and decreases for higher temperatures. Nevertheless, it can be noted that the value of the 
classical approximation becomes slightly larger (with a relative difference smaller than 0.1 per cent) than the direct sum at $\mathrm{T}=500 \mathrm{~K}$.

This reveals that, at any temperature above, when this difference will become substantially larger, the convergence of the direct sum obtained with the present spectroscopic data will not be reached anymore.

The values of the harmonic approximation of the vibrational partition function are given in Table 2 together with the rovibrational ones calculated through equation (4). The vibrational partition function is worked out using the fundamental vibrational frequencies determined with the $a b$ initio $\operatorname{CCSD}(\mathrm{T}) / \mathrm{cc}$-pCVTZ level of theory (Mladenovic et al. 2009). To calculate the rovibrational partition function, the values of the rotational direct sum are considered up to $\mathrm{T}=437 \mathrm{~K}$ inclusive since the direct sum provides larger values than those of the classical approximation. Above this temperature, the classical approximation is considered in its stead. In addition, in Table 2, the internal partition function of this work is set side by side with the values of the rotational partition function reported in the CDMS up to $\mathrm{T}=300 \mathrm{~K}$ (Endres et al. 2016). It should be highlighted that the relative difference is larger than 10 per cent at the temperature of $150 \mathrm{~K}$. In any way, a more precise partition function could be determined in case that new experimental spectral measurements as well as experimental fundamentals are available.

In the supplementary material, it is reported the rotational (as a direct sum up to $\mathrm{T}=437 \mathrm{~K}$ and from this temperature on as a classical approximation), vibrational, and rovibrational partition function computed in the present work for isofulminic acid (HONC) up to $\mathrm{T}=500 \mathrm{~K}$ using a $1 \mathrm{~K}$ interval.

This paper has been typeset from a $\mathrm{T}_{\mathrm{E}} \mathrm{X} / \mathrm{L} \mathrm{T}_{\mathrm{E}} \mathrm{X}$ file prepared by the author. 